\title{
Analyzing and Validating the Economic Efficiency of Managing a Cluster of Energy Hubs in Multi Carrier Energy Systems
}

\author{
Chen, Yue; Wei, Wei; Liu, Feng; Wu, Qiuwei; Mei, Shengwei
}

Published in:

Applied Energy

Link to article, DOI:

10.1016/j.apenergy.2018.08.112

Publication date:

2018

Document Version

Early version, also known as pre-print

Link back to DTU Orbit

Citation (APA):

Chen, Y., Wei, W., Liu, F., Wu, Q., \& Mei, S. (2018). Analyzing and Validating the Economic Efficiency of Managing a Cluster of Energy Hubs in Multi Carrier Energy Systems. Applied Energy, 230, 403-416.

https://doi.org/10.1016/j.apenergy.2018.08.112

\section{General rights}

Copyright and moral rights for the publications made accessible in the public portal are retained by the authors and/or other copyright owners and it is a condition of accessing publications that users recognise and abide by the legal requirements associated with these rights.

- Users may download and print one copy of any publication from the public portal for the purpose of private study or research.

- You may not further distribute the material or use it for any profit-making activity or commercial gain

- You may freely distribute the URL identifying the publication in the public portal 


\title{
Analyzing and Validating the Economic Efficiency of Managing a Cluster of Energy Hubs in Multi-Carrier Energy Systems
}

\author{
Yue Chen ${ }^{\mathrm{a}}$, Wei Wei ${ }^{\mathrm{a}}$, Feng $\mathrm{Liu}^{\mathrm{a}}$, Qiuwei $\mathrm{Wu}^{\mathrm{b}}$, Shengwei Mei ${ }^{\mathrm{a}}$ \\ ${ }^{a}$ State Key Laboratory of Control and Simulation of Power Systems and Generation Equipment, \\ Department of Electrical Engineering, Tsinghua University, 100084, Beijing, China. \\ ${ }^{b}$ Department of Electrical Engineering, Technical University of Denmark, Lyngby, 2800, Denmark
}

\begin{abstract}
The interdependency across natural gas, power and heating systems is increasingly tightened due to the wide development of cogeneration plants and electrified heating facilities. Multi-energy integration is a prevalent trend and the energy hub, which acts as an intermediary agent between providers and consumers, is expected to play a central role in allocating energy resources more efficiently. However, uncertainties originating from multiple kinds of energy demands challenge the operation of energy hubs and may compromise system efficiency. Energy trading and sharing among individual hubs offer a unique opportunity to increase system flexibility and reduce the cost under demand uncertainty. In this paper, three quintessential schemes for organizing a cluster of energy hubs at demand side, i.e., individual, sharing market, and aggregation, are studied under a stochastic framework with probabilistic load forecasts. First, we perform theoretical analysis and compare their economic efficiencies from a maximum-utility (or minimum-cost) perspective. Utility curves of respective schemes are given, and several important phenomena are revealed from the economic analysis. Then we discuss the concrete decision-making models of energy hubs under the three schemes, taking into account the change of electricity price in response to the total demand, which give rise to bilevel optimization problems and are technically transformed into mixed-integer linear programs. Finally, we conduct numerical experiments, which val-
\end{abstract}

\footnotetext{
*Corresponding author

Email address: l feng@tsinghua. edu. cn (Feng Liu)
} 
idate the theoretical outcomes, and reveal that the sharing scheme can achieve nearly optimal efficiency without a central organizer, and hence appears to be a promising direction for future multi-energy systems.

Keywords: Demand uncertainty, energy hub, energy-sharing market, multi-carrier energy system, organization scheme, stochastic bilevel game.

\title{
Nomenclature
}

\author{
Indices and Sets \\ $i \quad$ Index of energy hubs \\ $t \quad$ Index of time periods \\ $\omega \quad$ Index of scenarios \\ $j \quad$ Index of power generators \\ $n \quad$ Index of buses in power system
}

Parameter

$N_{E} \quad$ The number of energy hubs

$\eta_{e h} \quad$ Efficiency of electricity to heat conversion

$\eta_{g h} \quad$ Efficiency of gas to heat conversion

$\eta_{g e} \quad$ Efficiency of gas to electricity conversion

$\lambda_{t}^{e c}, \lambda_{e} \quad$ Electricity price in the day-ahead market

$\lambda_{t}^{g c}, \lambda_{g} \quad$ Natural gas price in the day-ahead market

$\lambda_{t, \omega}^{M}, \lambda_{M} \quad$ Gas-to-power price in the sharing market

$r_{m n}, x_{m n} \quad$ Resistance/Reactance of line $m n$

$\underline{v}_{n}, \bar{v}_{n} \quad$ Bounds of voltage magnitude square at bus $n$

$\underline{P}_{j}, \bar{P}_{j} \quad$ Bounds of active output of unit $j$

$\underline{Q}_{j}, \bar{Q}_{j} \quad$ Bounds of reactive output of unit $j$

$\bar{l}_{m n} \quad$ Square current capacity of line $m n$

$p_{n}^{l}, q_{n}^{l} \quad$ Active/reactive electricity demand at bus $n$

$c_{j} \quad$ Cost of generation unit $j$ 


$\begin{array}{ll}\pi, \pi_{\omega} & \text { Probability of each scenario } \\ \bar{I} & \text { Budget of the energy hub } \\ d_{e}, \tilde{l}_{i t, \omega}^{e} & \text { Real-time power demand } \\ d_{h}, \tilde{l}_{i t, \omega}^{h} & \text { Real-time heat demand } \\ E_{m}, H_{m} & \text { The capacity of power/heat storage unit } \\ R_{p m}^{ \pm}, R_{h m}^{ \pm} & \text {Maximum charge/discharge rate of storage unit } \\ M & \text { A large enough constant } \\ \left(p_{s}, \lambda_{s}\right) & \text { Breakpoints in piecewise linear technique }\end{array}$

\section{Decision Variable}

$\theta$

The expenditure of power

$\rho_{i t}$

Proportion of power converted into heat

$p_{i t}^{e, i n}, p_{i t}^{g, i n}$

Input electricity/gas of the energy hub

$p_{\text {it }}^{e, \text { out }}, p_{i t}^{h, \text { out }}$

Output electricity/heat of the energy hub

$p_{i t}^{d i s}, p_{i t}^{c h}$

Discharge/charge rate of power storage unit

$h_{i t}^{\text {dis }}, h_{i t}^{c h}$

Discharge/charge rate of heat storage unit

$\lambda_{t, \omega}^{e r}, \lambda_{t, \omega}^{h r}$

Price of electricity/heat in the real-time market

$u_{i t}, s_{i t}$

Binary variables indicate the status of storages

$E_{t}, H_{t}$

Energy amount of power storage/heat storage

$p_{i t}^{e 0}, p_{i t}^{g 0}$

Contracted power/gas in day-ahead market

$\delta_{i t, \omega}^{e+}, \delta_{i t, \omega}^{h+}$

Power/heat bought from the real-time market

$\delta_{i t, \omega}^{e-}, \delta_{i t, \omega}^{h-}$

Power/heat sold to the real-time market

$p_{i t, \omega}^{e x}$

Energy exchange in the sharing market

$p_{j}, q_{j}$

The active/reactive output of power generator $j$

$l_{m n}$

Square of the current in line $m n$

$v_{n}$

Square of voltage magnitude at bus $n$

$P_{m n}$

Active power flow in line $m n$

$Q_{m n}$

Reactive power flow in line $m n$

$\lambda_{n}$

Dual variable of the power balancing condition 
$\alpha_{s}$ Continuous weight variables used in piecewise linear technique

$\beta_{s} \quad$ Binary variables used in piecewise linear technique

$\gamma_{s} \quad$ Auxiliary variables used in objective function linearization

\section{Introduction}

Due to the synergy among different energy carriers [1], traditionally independently operated energy infrastructures such as the power grid, heating system, and natural gas system are now becoming increasingly interdependent because of the proliferation of co-generation plants, energy conversion devices, and energy storage units. Gas-fired combined heat and power (CHP) units have been proved to be more efficient compared with the separate production (an illustrative example can be found in [2]). The stateof-art air-source/ground-source heat pumps have a coefficient-of-performance (COP) up to 3-5 [3], which means that 3-5 times thermal energy can be extracted by consuming merely one unit amount of electricity. Although electric boilers have relative lower electricity-heat efficiency, they are very cheap to deploy and can absorb excessive renewable generation which is otherwise curtailed. In Europe, nearly $11 \%$ of its electricity was generated via cogeneration [4] while Demark, the Netherlands and Finland are the world's most intensive cogeneration economies [5]. In Germany, over 50\% of the nation's total electricity demand could be provided by cogeneration and it aims to double the share of cogeneration by 2020 [6]. In the UK, there has been a trend towards "multi-utility" bundling [7], increasing the coupling of multiple energy markets. CHP is already an important resource for the United State and constitutes $8 \%$ of generation capacity [8]. In this regard, the interdependence across multiple energy infrastructures will become more prevalent, especially in the countries/regions with long cold winter, creating strong interdependency in energy flow and market behavior [9].

Coordinated operation of multi-carrier energy systems has become a hot topic in recent years. The flexibility of combined heat and power system with thermal storage was evaluated based on a generic model in [10]. The energy flow of combined cooling heating and power system was analyzed under electrical demand management mode and thermal demand management mode respectively in [11]. The efficiency of separate 
operation and combined operation of heat and power production were compared in [12]. In many researches mentioned above, an implicit assumption is that a central operator has the authority to control components in all related systems. However, in current practice, different energy systems are usually owned or governed by individual entities, which may be unwilling to accept compulsory dispatch orders. In this regard, energy markets turn out to play an important role in allocating resources in a fairer way, since individual market participants can make decisions regarding their own purposes. The modeling and strategic planning methods of sustainable interdependent networks were presented in [13], where typical application examples can also be found.

The power market has been studied for decades. One classic organization is the pool-based market with the locational marginal price (LMP) scheme [14]. Traditional power market appears at the transmission level, and the market clearing comes down to a direct-current (DC) optimal power flow (OPF) problem [15]. Smart grid technologies allow the similar paradigm to be implemented in distribution systems. However, because the resistance to reactance ratio $(r / X)$ of distribution lines is higher than that in transmission grids, the alternating current (AC) OPF model is used to clear the distribution market [16]. The gas market is much less competitive and flexible than the electricity market. In the gas spot market, the price tends to be proportional to the gas demand and usually remains unchanged throughout a day [17]. To study the strategic interactions among multiple stakeholders in the gas market, a generalized Nash-Cournot game model was proposed in [18], and complementarity programming models were developed in [19] which were applied in South Stream [20]. As we restrict our attentions on the intra-day transaction, the gas price is assumed to be fixed as in [21].

With the increasingly tightened coupling of energy systems with multiple carriers, the advent of integrated energy markets will greatly promote energy transaction and sharing among different physical systems. Along this line of research, the market power of natural gas producers on the power market was investigated in [22]. A multilateral trading model for the gas-heat-power coupled system was proposed in [23] and the market behaviors of different energy systems were considered. In [24], a strategic offering model for the gas-power system was presented. The gas market is cleared in a similar way as the power market. All the studies above consider the real-time market 
without uncertainty. However, in the day-ahead market, the uncertain factors such as load forecast errors can no longer be neglected and may affect the real-time decisions.

In power market analysis, the impacts of load and price uncertainties have been investigated. Because of the competition and strategic behaviors of individual market participants, incorporating uncertainty in a market equilibrium model is much more difficult than doing so in a centralized dispatch problem. For the supply-side power market, a robust Cournot-Bertrand model was proposed in [25] to mimic risk-averse bidding strategies of generation companies in a congested power grid. Reference [26] proposed a day-ahead decentralized energy trading algorithm for power grid with generation uncertainty. A robust Nash game model was suggested in [27] to describe the market equilibrium of hydro-dominated power systems with strategic producers and water inflow uncertainty. For the demand-side market, the retailer is expected to play an active role. A stochastic optimization method was used in [28] to optimize the medium-term decision making for a power retailer. Uncertainty of future pool prices, client demands, and rival-retailer prices are taken into account. A stochastic multiobjective Nash-Cournot model was adopted in [29] to simulate the demand response under load uncertainty. A game based linear approach for retailer's scheduling under uncertain pool prices and load demands was presented in [30]. The scenario generation method and conditional value at risk $(\mathrm{CVaR})$ are used for risk measurement. Bilateral contracting and selling price determination problem for an electricity retailer under load uncertainty was proposed in [31]. Three pricing cases are compared, including the fixed pricing, time-of-use pricing and real-time pricing. The bidding and pricing strategies of retailer under market price uncertainty was studied in [32] using a robust bilevel programming model.

In a multi-energy system, energy hub is a new concept and initiatively related with multi-carrier energy linking [33]. An energy hub can be modeled as a multi-input multi-output prosumer which can receive, send, convert and store different types of energies [34]. In this sense, other energy system integration concepts with multiple energy inputs and multiple energy outputs can be described as a sort of energy hubs. For example, reference [35] modeled the networked micro-grids using the concept of multi-carrier energy hub, while an energy hub model was used to describe interactions 

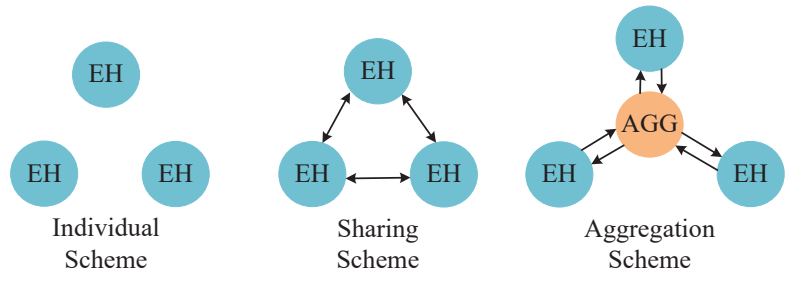

Figure 1: Sketch of three organization schemes.

among multiple energy systems in [36]. Application of the energy hub concept for the description of tri-generation devices was reported in [37]. Models for integrated energy and transportation systems were presented in [38]. The energy hub idea was also picked up by a municipal utility in Switzerland to model a micro-system containing wood chip gasification, methanation and a cogeneration plant [33]. After years of wonderful research, deterministic decision making for the energy hub dispatch problem has become a relatively mature field. Some works incorporating uncertainties have been carried out. For example, the decision-making problem of multi-energy players participating in the electricity market considering uncertain renewable energies was modeled in [39]. A stochastic bi-level model for energy hub manager was developed in [40]. The hub manager seeks to maximize its profit by selling electricity and heat under uncertain pool prices and load uncertainty.

A main research focus of energy hub is the modeling method to enable the incorporation of different energy facilities. This issue has been studied in the existing literature by using the automatic construction procedure [41], the block schematic diagram method [42], the decoupling method [43] and the linearized modeling method [36], to name a few. Another important problem is the business model of energy hubs taking their self-regarding behaviors into account, calling for a thorough study on trading scheme design, which is exactly the main motivation of this paper. The real-time scheduling of energy hubs in a dynamic pricing market was modeled as an exact potential game in [44] and an online distributed algorithm was proposed to find the Nash Equilibrium (NE). The existence and uniqueness of the NE was also investigated.

Different from aforementioned studies, which aim to provide optimal strategies for participants in a market, this paper investigates the economic efficiencies of three 
typical organization schemes, i.e., individual scheme, sharing scheme and aggregation scheme, of a cluster of energy hubs from a maximum-utility (or minimum-cost) perspective. A visual representation of three typical organization schemes is shown in Figure 1. Specifically, we consider an integrated energy system with electricity, natural gas as well as heat. The proposed model describes a bilevel trading framework which allows allocating a certain portion of resources in the upper level and coping with uncertainty in the lower level. Day-ahead and real-time markets are the most common and mature ones in the current electricity industry, and therefore are studied in this paper. The proposed method can be easily extended to problems with different time scales. The main contributions of this paper are twofold.

1) Theoretical analysis on the economic efficiencies of three typical energy-hub organization schemes. The economic efficiencies of individual, sharing, and aggregation schemes for energy transaction are examined from a maximum-utility (or minimum-cost) perspective under inaccurate load forecasts. Under reasonable assumptions, we prove that the aggregation scheme is the most efficient while the individual scheme is the most inefficient due to the lack of flexibility in real-time energy exchange. By allowing real-time energy transaction restricted by a fixed exchanging rate, which serves as the price in the sharing market, the efficiency of sharing scheme is in-between, but very close to the aggregation scheme, implying its promising perspective. The price in the sharing market is also revealed.

2) Concrete mathematical formulation of the three market schemes in a demandside integrated energy system and characterization of the market equilibrium. First, a bilevel framework of the studied system is set forth. In the pool-based market level, an ACOPF model is used to clear the distribution power market and retrieve the electricity price while the natural gas price and heat price are assumed as fixed values. In the demand-side integrated energy system level, stochastic optimization is employed to model the strategic behaviors of individual energy hubs. Aforementioned organization schemes are modeled via different constraints on energy exchange and allocation. To identify the market equilibria, the mathematical models are reformulated as mixed-integer linear programs (MILPs) based upon 
duality theory, linearization techniques and two properties of potential game and cooperative game. Finally, the proposed models are used to test and quantify the performance gaps of the three schemes using typical system data. Our method provides a systematic approach to formulating and efficiently computing equilibria of multi-energy markets.

The rest of this paper is organized as follows. Theoretical analysis is given in Section 2 to compare the economical efficiencies of the three energy hub organization schemes. The practical implementation and mathematical formulations of the energy hub decision-making under three organization schemes are presented in Section 3. Solution methodology is developed in Section 4. Case studies are performed in Section 5. Finally, conclusions are drawn in Section 6.

\section{Economical Efficiency: Theoretical Analysis}

Starting from a abstract formulation, we present a theoretical analysis on the efficiency of different organization schemes in the integrated energy systems with electricity, natural gas, and heat. Energy hubs participate in the day-ahead power and gas markets. At the supply side, electricity and natural gas are bought at prices $\lambda_{e}$ and $\lambda_{g}$, respectively. At the demand side, electricity and heat are needed. $d_{e}$ refers to the realtime power demand, which is a scalar and its SI unit is Watt. When the energy hub is going to sign a contract in the day-ahead market, it is not sure about the exact demands of its customers in the future. Here, system efficiency refers to the expected utility under demand uncertainty. The utility function can be defined as the profit function, which is the difference between incomes and costs.

For the sake of conciseness and illustration, we consider two extreme situations in real time: all the consumers need electricity with a probability of $\pi$ and all the consumers need heat with a probability of $1-\pi$. For the in-between cases where both power and heat are needed, the conclusion still holds. The utilities of the energy hub when it supplies electricity and heat are $u_{1}\left(d_{e}\right)$ and $u_{2}\left(d_{h}\right)$, which are concave, monotonically increasing, and differentiable [45]. Concavity and monotonicity are mild assumptions for utilities of smart grid users, as suggested in [46], such as the 
quadratic utility functions used in [45] and the logarithmic utility functions used in [47]. The energy hub allocates its budget on electricity and natural gas to maximize its expected utility,

$$
U\left(d_{e}, d_{h}\right)=\mathbb{E}[u]=\pi u_{1}\left(d_{e}\right)+(1-\pi) u_{2}\left(d_{h}\right)
$$

which is also concave. The contour line of the utility is a curve define as $U\left(d_{e}, d_{h}\right)=c$ where $c$ is a constant. We write the contour line as an explicit function,

$$
d_{h}=f_{\text {cont }}\left(d_{e}\right)=u_{2}^{-1}\left(\frac{c-\pi u_{1}\left(d_{e}\right)}{1-\pi}\right)
$$

Proposition 1. The contour line $d_{h}=f_{\text {cont }}\left(d_{e}\right)$ is a convex function which is decreasing in the first quadrant.

The monotonicity is clear: because $\pi u_{1}\left(d_{e}\right)$ is increasing in $d_{e}$ and $(1-\pi) u_{2}\left(d_{h}\right)$ is increasing in $d_{h}$, and their summation is a constant $c$, the growth in $d_{e}$ must lead to the decrease in $d_{h}$. To see the convexity, as $u_{1}\left(d_{e}\right)$ is concave, function

$$
g\left(d_{e}\right)=\frac{c-\pi u_{1}\left(d_{e}\right)}{1-\pi}
$$

is convex. Moreover, since $u_{2}\left(d_{h}\right)$ is concave and increasing. Its inverse function $u_{2}^{-1}$ is also increasing with a positive second-order derivative given by,

$$
\left(u_{2}^{-1}\right)^{\prime \prime}=-\frac{u_{2}^{\prime \prime}}{\left(u_{2}^{\prime}\right)^{2}}>0
$$

It implies $u_{2}^{-1}$ is convex. According to the composition law ([48], page 84), function $d_{h}=f_{\text {cont }}\left(d_{e}\right)$ is also convex.

Assume the energy hub has a total budget of $\bar{I}$, which is a constant scalar, and $\theta$ is spent on buying power and the rest on natural gas. The energy hub can produce heat from electricity with an efficiency of $\eta_{e h}$, and can convert natural gas into electricity and heat with efficiencies of $\eta_{g e}$ and $\eta_{g h}$, respectively. It is reasonable to make the following assumptions.

A1. Buying electricity directly is better than buying gas and producing electricity from gas, i.e.,

$$
\frac{1}{\lambda_{e}} \geq \frac{\eta_{g e}}{\lambda_{g}}
$$


A2. Both gas and electricity have none-zero contracts in day-ahead markets.

The day-ahead gas/electricity contract might be zero, if the day-ahead price is much higher than that in the real-time market or buying one kind of energy is more favorable. However, in such situations, the problem becomes much easier: if both day-ahead contracts are zero, since no uncertainty exists at the real-time stage, the energy hub can exactly determine the amount of each kind of energy according to the real demands, and exchange is not needed; if the day-ahead gas (electricity) contract is zero, which means all energy hubs buy gas (electricity) only, there is no exchange either. Therefore, the problem degenerates into a trivial one and we simply exclude this trivial case by assuming A2.

Because energy hub can switch the usage between electricity and natural gas, implying the potential of energy sharing among different energy hubs, it is interesting to study how the utilities of individual energy hubs can be improved by allowing energy exchange. Here, we analyze three emblematic energy hub organization schemes:

1) Individual scheme (IDL): each energy hub makes its decision respectively and no trading among energy hubs is allowed. Operation of energy hubs following this scheme has been discussed in the medium-term management problem [49] and the intra-day decision-making problem [50].

2) Sharing market scheme (SMK): an energy hub can exchange energy with others at a fixed ratio $\lambda_{M}$, which is called the price in the sharing market. In fact, it is not a financial concept with a monetary unit but a barter ratio. It defines how much electricity can be exchanged with one unit natural gas. In this regard, the sharing market is a platform for energy sharing without monetary transaction. Operation of networked microgrids following this scheme can be found in [51], and a decentralized energy exchange strategy for prosumers in smart grid was presented in [52].

3) Aggregation scheme $(A G G)$ : a central aggregator (or retailer) is eligible to collect and redistribute energy among all energy hubs. This scheme has been discussed under the concept of multi-energy player in electricity markets [40] and in local energy system [53].

Next we analyze the three organization schemes theoretically. 


\section{(1) Individual (IDL)}

In this case, the total budget $\bar{I}$ is allocated to electricity with the amount of $\theta$ and natural gas with $\bar{I}-\theta$. In the all power demand situation, the electricity supply is,

$$
d_{e}=\frac{\theta}{\lambda_{e}}+\frac{\bar{I}-\theta}{\lambda_{g}} \cdot \eta_{g e}
$$

where the first term represents electrical power that is directly purchased from the power grid, and the second one stands for which is produced by the CHP unit. In the all heat demand situation, the heat supply is,

$$
d_{h}=\frac{\theta}{\lambda_{e}} \cdot \eta_{e h}+\frac{\bar{I}-\theta}{\lambda_{g}} \cdot \eta_{g h}
$$

where the first term represents heat that is converted from the electrified heating devices, and the second one stands for heat produced by the CHP unit.

The optimal decision in the IDL scheme is shown in Figure 2 If $\theta=0$, then $d_{e}=\eta_{g e} \bar{I} / \lambda_{g}$ and $d_{h}=\eta_{g h} \bar{I} / \lambda_{g}$; if $\theta=\bar{I}$, then $d_{e}=\bar{I} / \lambda_{e}$ and $d_{h}=\eta_{e h} \bar{I} / \lambda_{e}$; otherwise when $\theta$ constantly changes from 0 to $\bar{I}$, the point $\left(d_{e}, d_{h}\right)$ forms a line segment $\mathbf{a b}$. The contour line of $U\left(d_{e}, d_{h}\right)$ is also shown in Figure 2, whose shape has been revealed in Proposition 1. We can observe that the optimal choice of an energy hub is to buy electricity as long as Assumption $\mathrm{A} 1$ holds.

\section{(2) Sharing market (SMK)}

In the sharing market, energy hubs can trade with each other to meet energy demands. Assume that $\lambda_{M}$ is the price (exchange ratio) at equilibrium, then the energy supply in the two extreme situations are shown as,

$$
\left\{\begin{array}{l}
d_{e}=\frac{\theta}{\lambda_{e}}+\max \left\{\frac{\bar{I}-\theta}{\lambda_{g}} \lambda_{M}, \frac{\bar{I}-\theta}{\lambda_{g}} \eta_{g e}\right\} \\
d_{h}=\max \left\{\frac{\theta}{\lambda_{e}} \eta_{e h}, \frac{\theta}{\lambda_{e}} \frac{1}{\lambda_{M}} \eta_{g h}\right\}+\frac{\bar{I}-\theta}{\lambda_{g}} \eta_{g h}
\end{array}\right.
$$

Take the electricity demand for example, the first term represents electrical power that is directly purchased from the power grid, and the rest can be obtained in two ways: one is to trade with other hubs in the sharing market, and the other is to dispatch the CHP. The second term picks up the bigger one in the curly braces. The latter equation in (5) has a similar interpretation. To analyze the sharing market, we give the following Lemma. 
Lemma 1. Assume Assumption A 1 and A 2 hold, we have,

$$
\frac{\eta_{e h}}{\lambda_{e}} \leq \frac{\eta_{g h}}{\lambda_{g}} ; \quad \lambda_{M}=\frac{\lambda_{g}}{\lambda_{e}} ; \quad d_{e}=\frac{\bar{I}}{\lambda_{e}} ; \quad d_{h}=\frac{\bar{I}}{\lambda_{e}} \eta_{g h}
$$

Proof. First of all we consider the first inequality. For the sake of contradiction, assume

$$
\frac{\eta_{e h}}{\lambda_{e}}>\frac{\eta_{g h}}{\lambda_{g}}
$$

It implies that producing heat from electricity is cheaper than using natural gas. Moreover, as indicated by (2), purchasing electricity from the power grid has a lower cost than buying gas and using CHP units. As a result, all energy hubs will consume electricity, and natural gas will lose all market share, which is in contradiction with Assumption A2. So

$$
\frac{\eta_{e h}}{\lambda_{e}} \leq \frac{\eta_{g h}}{\lambda_{g}}
$$

must hold.

Combined with (2), we have,

$$
\eta_{g e} \leq \frac{\lambda_{g}}{\lambda_{e}} \leq \frac{\eta_{g h}}{\eta_{e h}}
$$

Next we consider the second assertion. The following discussion is divided into three cases.

1) If $\lambda_{M} \leq \eta_{g e}$, with (7) we have $\eta_{g h} / \lambda_{M} \geq \eta_{e h}$. Hence the max qualifier in (5) can be dropped and the energy demands in the two extreme situations can be expressed as,

$$
\left\{\begin{array}{l}
d_{e}=\frac{\theta}{\lambda_{e}}+\frac{\bar{I}-\theta}{\lambda_{g}} \eta_{g e} \\
d_{h}=\frac{\theta}{\lambda_{e}} \frac{\eta_{g h}}{\lambda_{M}}+\frac{\bar{I}-\theta}{\lambda_{g}} \eta_{g h}
\end{array}\right.
$$

We can conclude from (7p) that $\lambda_{M} \leq \lambda_{g} / \lambda_{e}$. And then we have

$$
\frac{\eta_{g h}}{\lambda_{e} \lambda_{M}} \geq \frac{\eta_{g h}}{\lambda_{g}}
$$

which indicates that purchasing electricity from the power grid and trading gas in the sharing market is a better way than buying natural gas directly for producing heat. So all energy hubs will buy electricity and no gas contract will be signed, contradicting Assumption A 2 
2) If $\lambda_{M}>\eta_{g e}$ and $\eta_{g h} / \lambda_{M} \geq \eta_{e h}$, then the energy demands in the two extreme situations can be expressed by,

$$
\left\{\begin{array}{l}
d_{e}=\frac{\theta}{\lambda_{e}}+\lambda_{M} \frac{\bar{I}-\theta}{\lambda_{g}} \\
d_{h}=\frac{\theta}{\lambda_{e}} \frac{\eta_{g h}}{\lambda_{M}}+\frac{\bar{I}-\theta}{\lambda_{g}} \eta_{g h}
\end{array}\right.
$$

In such a circumstance, if

$$
\lambda_{M}>\frac{\lambda_{g}}{\lambda_{e}} \text { or } \frac{1}{\lambda_{g}}>\frac{1}{\lambda_{e} \lambda_{M}}
$$

which means that signing a contract with the gas market can get more natural gas than buying electricity and then trading gas in the sharing market with the same amount of expenditure. As a consequence, all energy hubs will not buy electricity. On contrary, no one will buy natural gas. This is in contradiction with the assumption. So we have,

$$
\lambda_{M}=\frac{\lambda_{g}}{\lambda_{e}}
$$

3) If $\lambda_{M}>\eta_{g e}$ and $\eta_{g h} / \lambda_{M}<\eta_{e h}$, then the energy demands in the two extreme situations can be written as,

$$
\left\{\begin{array}{l}
d_{e}=\frac{\theta}{\lambda_{e}}+\lambda_{M} \frac{\bar{I}-\theta}{\lambda_{g}} \\
d_{h}=\frac{\theta}{\lambda_{e}} \eta_{e h}+\frac{\bar{I}-\theta}{\lambda_{g}} \eta_{g h}
\end{array}\right.
$$

In such a circumstance, we can conclude that $\lambda_{M}>\lambda_{g} / \lambda_{e}$ owing to the second inequality of (7), which is the same to the case we have discussed in Item 2), in which all energy hubs will buy gas and no electricity contract will be signed.

In summary, $\lambda_{M}=\lambda_{g} / \lambda_{e}$ must hold, implying assertion 2) holds.

Substituting it into 97 yields

$$
d_{e}=\frac{\bar{I}}{\lambda_{e}}, d_{h}=\frac{\bar{I}}{\lambda_{e}} \eta_{g h}
$$

Then assertions 3) and 4) hold, which completes the proof.

Equation (11) interprets that an energy hub will purchase both electricity and heat, and exchange in sharing market depending on whether electricity or heat is needed. 
The optimal strategy of the energy hub in the sharing market is plotted as point $\mathbf{c}$ in Figure 2

\section{(3) Aggregation (AGG)}

In this organization scheme, a large number of energy hubs are coordinated by an aggregator, who is eligible to sign aggregated contracts with the power and gas markets on behalf of the energy hubs and redistribute energies among the clients. According to the Law of Large Numbers in the probability theory, the number of energy hubs which buy electricity (or natural gas) is $N \pi$ (or $N(1-\pi)$ ). Hence the energy demands satisfy,

$$
\left\{\begin{array}{l}
N \pi \cdot d_{e}=\frac{N \theta}{\lambda_{e}} \\
N(1-\pi) \cdot d_{h}=\frac{N(\bar{I}-\theta)}{\lambda_{g}} \eta_{g h}
\end{array}\right.
$$

From an average point of view, it is,

$$
d_{e}=\frac{\theta}{\pi \lambda_{e}}, d_{h}=\frac{(\bar{I}-\theta) \eta_{g h}}{(1-\pi) \lambda_{g}}
$$

The optimal decision in the aggregation mode is also shown in Figure 2 When $\theta$ varies from 0 to $\bar{I}$, the point $\left(d_{e}, d_{h}\right)$ forms the line segment de. The optimal solution will be found at point $\mathbf{f}$ where line de is tangent to a contour line of $U\left(d_{e}, d_{h}\right)$.

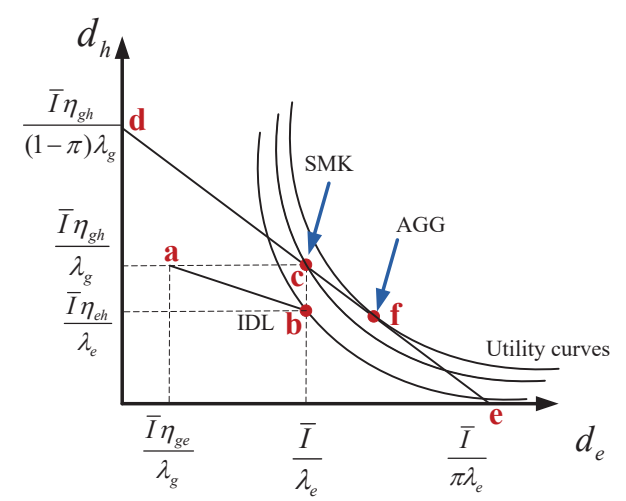

Figure 2: Optimal decisions under three organization modes.

With above analysis, we have the following proposition. 


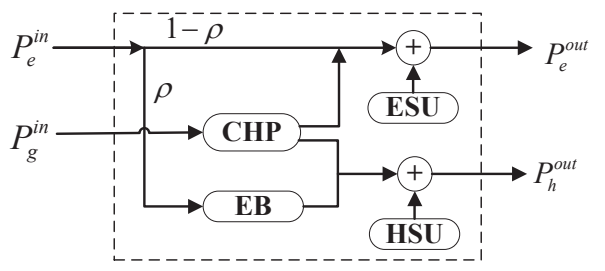

Figure 3: Structure of a sample energy hub.

Proposition 2. Let $E U_{I D L}, E U_{S M K}$, and $E U_{A G G}$ be the optimal expected utility $U\left(d_{e}, d_{h}\right)$ in the individual, sharing market, and aggregation schemes. There must be,

$$
E U_{I D L}<E U_{S M K}<E U_{A G G}
$$

The relationship of the three organization schemes can be observed from Figure 2. The optimal expected utilities are evaluated at points $\mathbf{b}$, $\mathbf{c}$, and $\mathbf{f}$. Because $U\left(d_{e}, d_{h}\right)$ is increasing in the two inputs, clearly we have $E U_{I D L}<E U_{S M K}$. Moreover, it is easy to verify that point $\mathbf{c}$ belongs to line segment $\mathbf{d e}$, and $U\left(d_{e}, d_{h}\right)$ perceives the largest value at point $\mathbf{f}$ when the pair $\left(d_{e}, d_{h}\right)$ varies in the line segment $\mathbf{d e}$. Therefore, we have $E U_{S M K}<E U_{A G G}$.

\section{Implementation in the Integrated Energy System}

The conclusion drawn in Proposition 2 is based on the abstract model without reflecting practical system configurations. For further investigation, we propose thorough mathematical formulations for an integrated energy system with a cluster of energy hubs, which can be modeled as stochastic bilevel problems. In the energy hub level, stochastic optimization models are adopted to analyze the energy hubs' strategic behaviors under three organization schemes. In the pool-based market level, an ACOPF model is used to retrieve the LMP. Detailed formulation of each parts are given in the following subsections.

\subsection{Energy Hub Model}

Energy hub is the substantial participant in the multi-carrier energy market. In this paper, we consider an energy hub with two inputs (electricity and gas) and two outputs 
(electricity and heat). Its structure is illustrated in Figure 3 . The import electricity can be transformed into heat by an electric boiler (EB), or be used to charge an electricity storage unit (ESU). Heat pumps can be modeled by the same way as the EB with a higher efficiency coefficient. Natural gas is burnt by a CHP unit which generates electricity and heat. The heat can be stored in a heat storage unit (HSU). Operating constraints include,

$$
\begin{gathered}
\left(1-\rho_{i t}\right) p_{i t}^{e, i n}+p_{i t}^{g, i n} \eta_{g e}+p_{i t}^{d i s}-p_{i t}^{c h}=p_{i t}^{e, o u t} \\
\rho_{i t} p_{i t}^{e, i n} \eta_{e h}+p_{i t}^{g, i n} \eta_{g h}+h_{i t}^{d i s}-h_{i t}^{c h}=p_{i t}^{h, o u t} \\
E_{i(t+1)}=E_{i t}+p_{i t}^{c h} \eta_{e s}^{+}-p_{i t}^{d i s} / \eta_{e s}^{-}, E_{i 0}=0 \\
H_{i(t+1)}=H_{i t}+h_{i t}^{c h} \eta_{h s}^{+}-h_{i t}^{d i s} \eta_{h s}^{-}, H_{i 0}=0 \\
0 \leq p_{i t}^{c h} \leq u_{i t} R_{p m}^{+}, 0 \leq p_{i t}^{d i s} \leq\left(1-u_{i t}\right) R_{p m}^{-} \\
0 \leq h_{i t}^{c h} \leq s_{i t} R_{h m}^{+}, 0 \leq h_{i t}^{d i s} \leq\left(1-s_{i t}\right) R_{h m}^{-} \\
0 \leq E_{i t} \leq E_{m}, 0 \leq H_{i t} \leq H_{m}
\end{gathered}
$$

where $\rho_{i t}$ represents the proportion of import electricity which is used for heat production. Constraints (13a)-13b] are electric power and thermal balancing conditions; 13c-13d describe charging dynamics of energy storage units; 13e-13f impose maximal charging and discharging rates on storage units as well as their complementarity; (13g) limits the energy stored in the ESU and the HSU. Although the first terms in (13a) and 13b) are bilinear, they can be replaced by two new variables $p_{i t}^{e, e}$ and $p_{i t}^{e, h}$ in combination with an equality constraint $p_{i t}^{e, e}+p_{i t}^{e, h}=p_{i t}^{e, i n}$. In this way, all constraints in (13) are linear. 


\subsection{Real-time Power Market Clearing Model}

We assume that the real-time electricity price is equal to the LMP released by a distribution power market. Because the resistance of distribution lines have notable impact on LMP, we use the following branch flow model [54] based AC-OPF for distribution power market clearing in the real-time stage

$$
\begin{aligned}
& \min \sum c_{j} p_{j} \\
& \text { s.t. } v_{m}-v_{n}=2\left(r_{m n} P_{m n}+x_{m n} Q_{m n}\right)-\left(r_{m n}^{2}+x_{m n}^{2}\right) l_{m n} \\
& l_{m n} v_{m}=P_{m n}^{2}+Q_{m n}^{2} \\
& p_{n}-p_{n}^{l}=\sum_{k: n \rightarrow k} P_{n k}-\left(P_{m n}-l_{m n} r_{m n}\right): \lambda_{n} \\
& q_{n}-q_{n}^{l}=\sum_{k: n \rightarrow k} Q_{n k}-\left(Q_{m n}-l_{m n} x_{m n}\right) \\
& \underline{P}_{j} \leq p_{j} \leq \bar{P}_{j}, \underline{Q}_{j} \leq q_{j} \leq \bar{Q}_{j} \\
& \underline{v}_{n} \leq v_{n} \leq \bar{v}_{n} ; 0 \leq l_{m n} \leq \bar{l}_{m n}
\end{aligned}
$$

where $c_{j}$ and $p_{j}$ are the cost coefficient and output of generation units, respectively. Objective function $114 \mathrm{a}$ is to minimize the operating cost. Constraints $14 \mathrm{~b}-1 \mathrm{14 \textrm {e }}$ state the branch flow model developed in [54], which provides the same solution as the traditional bus injection model for radial networks [55]. $p_{n}^{l}$ is the active power demand at bus $n$, including the fixed residential/industrial demand and elastic demand from energy hubs. Dual variable $\lambda_{n}$ associated with nodal active power balancing condition (14d) interprets the LMP. Physical limitations of generator output, bus voltage magnitudes, and line currents are included in constraints [14f]-(14g). We assume the real-time power market is cleared every period according to a single-period OPF problem, which is in compliance with the current power market practice. Hence we omit the time index in (14). The typical frequency is once per hour.

Remark 1: For the distribution power market, various entities can participate and bid their demands as shown in Figure 4 Multi-energy trading is restricted at the demand side. For clarity and simplification, this paper only considers the strategic behaviors of energy hubs and assumes that the bids of other participants are given as fixed nodal power demands. Demand response can be incorporated via a price-sensitive load 
with a price-demand function as is suggested in [56].

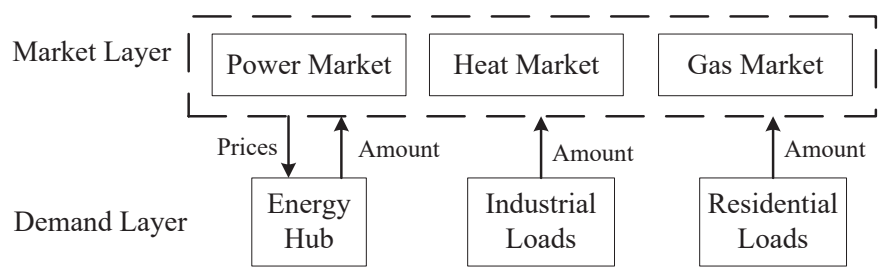

Figure 4: Structure of the bilevel multi-energy market.

\subsection{Holistic Market Configurations}

The structure of the integrated energy system is shown in Figure 5. In the dayahead market, the energy hubs determine their gas and power consumptions and sign forward contracts with the power market and natural gas market with fixed prices $\lambda_{t}^{e c}$ and $\lambda_{t}^{g c}$. At this time, the exact electricity and heat demands are not known exactly, and we have probabilistic forecasts on their possible values. When the exact load is revealed or can be predicted with high accuracy, energy hubs can buy (sell) energies from (to) the real-time markets. The real-time electricity price is given by the market clearing problem stated in (14). According to the current gas/heat market organization, the gas/heat price does not vary intraday, so $\lambda_{t}^{g c}$ and $\lambda_{t}^{h r}$ are equal to given values.

In addition, energy hubs could convert undesired energy into the desired form and exchange energies with other hubs. Three organization schemes are considered. For the individual scheme, energy hubs cannot trade with others, so they must satisfy real-time energy demands by energy conversion and energy purchase. For the sharing market scheme, energy hubs can exchange electricity and natural gas with other hubs subject to an exchange price/ratio. For the aggregation scheme, the retailer will collect and redistribute energies to all participants.

In analogy to the organization of current deregulated power markets, we consider two phases in the proposed organization scheme:

\section{Phase 1: Day-ahead stage}

Assume that the electricity price is $\lambda_{t}^{e c}$, and the gas price is $\lambda_{t}^{g c}$.

For IDL and SMK schemes, each energy hub decides on its contracted electricity 
$p_{i t}^{e 0}$ and gas $p_{i t}^{g 0}$. For AGG scheme, the aggregator will collect the demand from all energy hubs and decide the total contracted electricity $\sum_{i} p_{i t}^{e 0}$ and total contracted gas $\sum_{i} p_{i t}^{g 0}$.

\section{Phase 2: Real-time stage}

Assume that the electricity price is $\lambda_{t}^{e r}$ (LMP) and the gas price is $\lambda_{t}^{h r}$.

For IDL scheme, each energy hub buys/sells electricity $\delta_{i t, w}^{e \pm}$ and heat $\delta_{i t, w}^{h \pm}$ from/to the real-time market to meet load demand. For SMK scheme, each energy hub can exchange gas $p_{i t}^{e x}$ for power $p_{i t}^{e x} \lambda_{t, w}^{M}$ with other energy hubs at a fixed ratio $\lambda_{t, w}^{M}$, and buys/sells electricity $\delta_{i t, w}^{e \pm}$ and heat $\delta_{i t, w}^{h \pm}$ from/to the real-time market to meet load demand. For AGG scheme, the aggregator buys/sells electricity $\sum_{i} \delta_{i t, w}^{e \pm}$ and heat $\sum_{i} \delta_{i t, w}^{h \pm}$ from/to the real-time market and redistributes the energies to each hub to meet load demand.

\subsection{Formulation of the Market Equilibrium Problems}

In the integrated energy system, each energy hub tries to maximize (minimize) its own utility (payoff). The payoff function of energy hub $i$ has the following form

$$
\begin{aligned}
C_{i}^{H} & =\sum_{t}\left(\lambda_{t}^{e c} p_{i t}^{e 0}+\lambda_{t}^{g c} p_{i t}^{g 0}+\sum_{\omega=1}^{\Omega} \pi_{\omega}\left[-\lambda_{t, \omega}^{e r} \delta_{i t, \omega}^{e-}\right.\right. \\
& \left.\left.-\lambda_{t, \omega}^{h r} \delta_{i t, \omega}^{h-}+\lambda_{t, \omega}^{e r} \delta_{i t, \omega}^{e+}+\lambda_{t, \omega}^{h r} \delta_{i t, \omega}^{h+}+\operatorname{Pen}\left(\delta_{i t, \omega}^{h \pm}, \delta_{i t, \omega}^{e \pm}\right)\right]\right)
\end{aligned}
$$

The sum of the first and second terms denotes the cost of buying energies from the dayahead markets; the remaining terms describe the income and the payment in real-time electricity and district heating markets, which are scenario dependent. The last term is a penalty for being unable to fulfill the contract.

The energy transaction constraints are formulated as,

$$
\begin{aligned}
& \sum_{t}\left(\lambda_{t}^{e c} p_{i t}^{e 0}+\lambda_{t}^{g c} p_{i t}^{g 0}\right) \leq \bar{I} \\
& p_{i t, \omega}^{e, o u t}+\delta_{i t, \omega}^{e+}-\delta_{i t, \omega}^{e-}=\tilde{l}_{i t, \omega}^{e}, \forall t \\
& p_{i t, \omega}^{h, o u t}+\delta_{i t, \omega}^{h+}-\delta_{i t, \omega}^{h-}=\tilde{l}_{i t, \omega}^{h}, \forall t \\
& \delta_{i t, \omega}^{e+}, \delta_{i t, \omega}^{e-}, \delta_{i t, \omega}^{h+}, \delta_{i t, \omega}^{h-}, p_{i t}^{e 0}, p_{i t}^{g 0} \geq 0, \forall t
\end{aligned}
$$




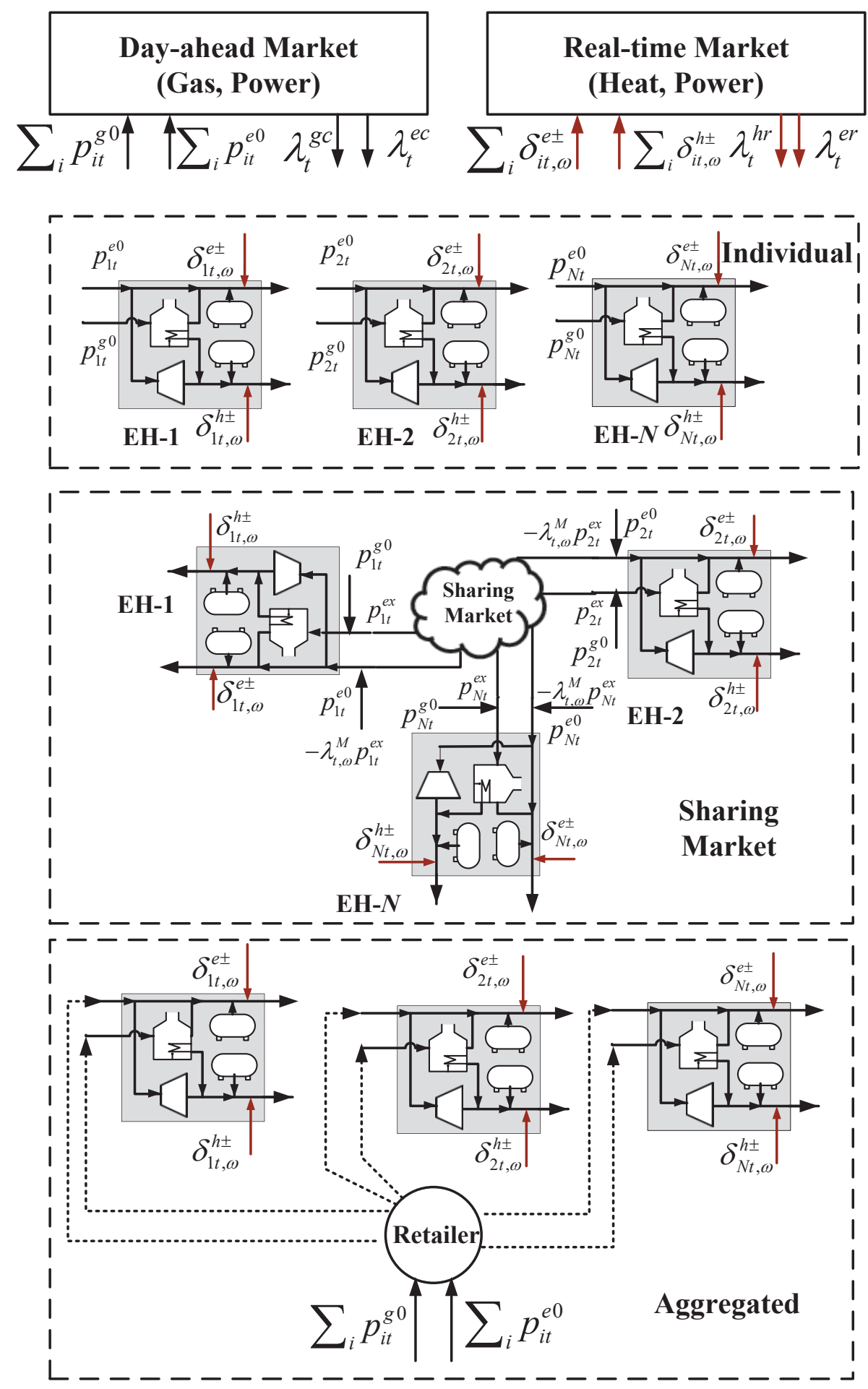

Figure 5: Structure of the integrated energy market. 
where $(16 \mathrm{a})$ is the budget limit in the day-ahead market; (16b) and $(16 \mathrm{c}$ ) are the realtime power and heat balance conditions; $16 \mathrm{~d}$ prevents negative values on decision variables.

As mentioned above, apart from energy transaction with the pool-based distribution market, the organization scheme of energy hubs also affects the economical efficiency. Next, we set forth the energy hub decision problems under the three organization schemes.

(1) Individual scheme. Under this setting, each energy hub makes decision individually and cannot trade with other hubs. In the real-time, they narrow the gap between forward contract and real demand by using conversion facilities, such as electric boilers, CHPs and storage units. The problem for each energy hub $i$ is shown below.

$$
\left.\begin{array}{ll}
\min & C_{i}^{H} \\
\text { s.t. } & \text { energy transaction constraints } 16] \\
& \text { hub operating constraints } 13] \\
& \text { market clearing condition (14), } \forall t \\
& p_{i t}^{e 0}=p_{i t, \omega}^{e, i n}, p_{i t}^{g 0}=p_{i t, \omega}^{g, i n}, \forall i, \forall t
\end{array}\right\}, \forall i
$$

The last constraint characterizes energy availability in the individual setting. Because energy exchange among hubs is prohibited, the import energies follow the forward contracts. Moreover, the real time electricity price $\lambda_{t}^{e r}$ is determined by the market clearing problem (14). Thus, problem (17) is in fact a bilevel program, whose solution strategy will be illuminated in the next section.

(2) Sharing market scheme. Under this setting, energy hubs have more flexibility because they can trade with each other. Let $\lambda_{t, \omega}^{M}$ be the sharing market price in scenario 
$\omega$ at period $t$. The problem for each energy hub $i$ is shown below

$$
\left.\begin{array}{ll}
\min & C_{i}^{H} \\
\text { s.t. } & \text { energy transaction constraints } 16] \\
& \text { hub operating constraints } 13 \text { (13) } \\
& \text { market clearing condition (14), } \forall t \\
& p_{i t}^{e 0}-p_{i t, \omega}^{e x} \lambda_{t, \omega}^{M}=p_{i t, \omega}^{e, i n}, \forall t \\
& p_{i t}^{g 0}+p_{i t, \omega}^{e x}=p_{i t, \omega}^{g, i n}, \forall t \\
& \sum_{i=1}^{N_{E}} p_{i t, \omega}^{e x}=0, \forall t
\end{array}\right\}, \forall i
$$

The constraints in $(18)$ are similar to those in $(17)$ except for the energy allocation conditions. In this setting, the imports of energy hubs come not only from the forward contracts but also from the energy exchanges in the sharing market. In addition, the total natural gas exchange in the system in every period should be balanced (so should the electricity), which is ensured by the last equality. Problem (18) can be transformed to a mathematical program with equilibrium constraints, which will be detailed in the next section.

(3)Aggregation scheme. Under this setting, the retailer/aggregator is responsible for collecting the requirements of all energy hubs and makes a centralized decision. The problem for the retailer is shown below,

$$
\begin{array}{ll}
\min & \sum_{i=1}^{N_{E}} C_{i}^{H} \\
\text { s.t. } & \text { energy transaction constraints (16), } \forall i \\
& \text { hub operating constraints (13), } \forall i \\
& \text { market clearing condition 14], }, \forall t, \forall i \\
& \sum_{i=1}^{N_{E}} p_{i t}^{e 0}=\sum_{i=1}^{N_{E}} p_{i t, \omega}^{e, \text { in }}, \sum_{i=1}^{N_{E}} p_{i t}^{g 0}=\sum_{i=1}^{N_{E}} p_{i t, \omega}^{g, \text { out }}, \forall t, \forall i
\end{array}
$$

Compared to problem (18), problem (19) minimizes the total payoff of all energy hubs subject to the Cartesian product of their feasible regions together with a system-level import energy constraint depending on the forward energy contracts.

It can be observed that the difference among problems $177-197$ mainly stems from 
the energy allocation conditions. The feasible region becomes more relaxed when the organization switches from individual to sharing market and moreover to aggregation.

\section{Solution Methodology}

The energy hubs' problems (17) and 18 in individual and sharing market schemes are multi-leader single-follower games, where the follower problem captures the market clearing process. The remaining one has a single retailer in the upper level, and comes down to a traditional bilevel program or a Stackelberg game. In this section, we first show the former two problems are actually potential games, so their objective can be aggregated into a single one for the ease of computation. Then we present MILP approximations for problems (17)-(19), which can be solved efficiently by commercial software. By exploiting the problem structure, our method does not rely on KKT optimality conditions, and is more efficient and scalable compared with existing methods for bi-level programs.

\subsection{Additivity of the Objective Functions}

In problem (17), although the objective functions are totally decoupled, the market clearing condition depends on the joint actions of all energy hubs. Therefore, the individual problems for each hub are still correlated. Nevertheless, the following proposition asserts that their objective functions can be added together, resulting in a traditional Stackelberg game.

Proposition 3. Problem (17) and the following Stackelberg game share the same equilibrium solution.

$$
\begin{array}{ll}
\min & \sum_{i=1}^{N_{E}} C_{i}^{H} \\
\text { s.t. } & \text { energy transaction constraints }[16, \forall i \\
& \text { hub operating constraints } 1 \text { 13] } \\
& \text { market clearing condition } 14, \forall t \\
& p_{i t}^{e 0}=p_{i t, \omega}^{e, i n}, p_{i t}^{g 0}=p_{i t, \omega}^{g, i n}, \forall i, \forall t
\end{array}
$$


According to the property of potential games [57], it is easy to verify that $f_{\text {atk }}=$ $\sum_{i}^{N_{E}} C_{i}^{H}$ is a potential function for problem (17). The result of single-level potential games has been generalized to bilevel games in [58]. The equilibrium consistency between a multi-leader common-follower game and its corresponding potential Stackelberg game is demonstrated by comparing their stationarity conditions. Proposition 3 is a straightforward application of the outcome therein. By such equivalence, we can also claim that problem (17) possesses a pure-strategy equilibrium as long as problem 20) has a feasible solution (since it must be bounded).

A similar conclusion holds for problem (18) and the following Stackelberg game:

$$
\begin{array}{ll}
\min & \sum_{i=1}^{N_{E}} C_{i}^{H} \\
\text { s.t. } & \text { energy transaction constraints }[16, \forall i \\
& \text { hub operating constraints }[13] \\
& \text { market clearing condition }[14], \forall t \\
& p_{i t}^{e 0}-p_{i t, \omega}^{e x} \lambda_{t, \omega}^{M}=p_{i t, \omega}^{e, i n}, \forall t, \forall i \\
& p_{i t}^{g 0}+p_{i t, \omega}^{e x}=p_{i t, \omega}^{g, i n}, \forall t, \forall i \\
& \sum_{i=1}^{N_{E}} p_{i t, \omega}^{e x}=0, \forall t, \forall i
\end{array}
$$

Since no competition exists in the upper levels of problems 20 and 21 , they are easier to solve than the original ones in $(17)$ and $(18)$. A problem remaining to be answered is: why will the energy hubs form a whole coalition? Is it possible that multiple smaller sharing markets exist at the same time?

Proposition 4. All the energy hubs will take part in a whole sharing market.

This proposition is a natural implication of the coalition based cooperative game theory. Suppose that there are two sub-markets, and if they could exchange energy with the each other following the rule of energy trading and clearing in $(18)$, then the total payoff must be no greater than the sum of respective sub-markets. That is because, two independent operating sub-markets is simply a special case of the whole sharing market, where the energy exchange between two sub-markets is prohibited. So the operating feasible region of the whole sharing market is enlarged. In this regard, the 
cooperation among energy hubs can be described as a super additive game, where the grant coalition must grab the highest profit (or the lowest cost) [59].

Standard approaches for solving a bi-level program relies on replacing the lowerlevel problem with its KKT optimality conditions. However, in problems (19)-(21), the market clearing problem in the lower level are non-convex, and KKT conditions are only necessary and remain computational challenging. In what follows, we will develop a decomposition method for coping with such an issue by exploiting the fact that only the LMP at a single bus is involved.

\subsection{Mixed Integer Linear Programming based Approximations}

To overcome the computation challenges brought by the lower-level market clearing problem and solve problems (19)-(21) in a systematic way, they are approximated by MILPs after performing the following three steps. The motivation of performing linearization is raised by observing the matter of fact: the majority of constraints are linear in our model. If we can linearize the objective function and the few nonlinear constraints generated by the primal-dual condition, the problem becomes an MILP which can be solved efficiently by CPLEX. The benefit is that the (approximate) global optimal solution can be found in reasonable time while a nonlinear model can only be solved locally.

\section{(1) Portraying the LMP curve}

Problem (14) is non-convex due to the quadratic equality $14 \mathrm{c}$. The convex relaxation method [54] is performed which replaces "=" in 14c with " $\geq$ ", as such the non-convex quality is turned into a rotated second-order cone. Sufficient conditions that guarantee the exactness of such a convex relaxation are revealed in [60], and are usually satisfied in most distribution systems with radial topology. Given the net injections, the relaxed problem, i.e., an second-order cone problem (SOCP), can be efficiently solved, and the LMP $\lambda$ at the connection bus can be retrieved from the dual variable associated with the nodal active power balancing equality in (14d). This function is supported in off-the-shelf solvers, such as MOSEK.

Changing the power demand at the connection bus over a desired interval, and recording the corresponding values of $\lambda$, we obtain the LMP-demand curve needed in 


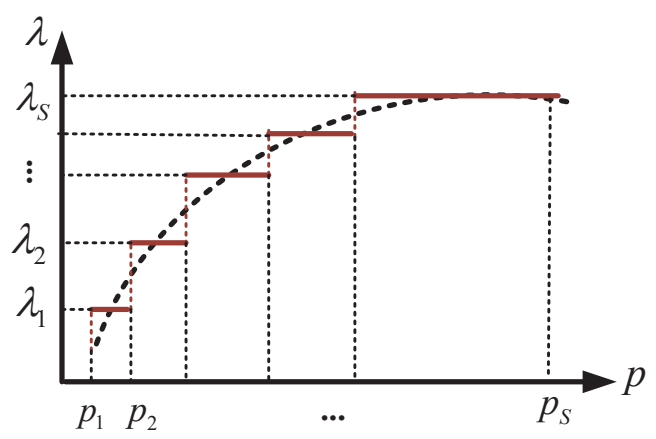

Figure 6: Piecewise constant approximation of the LMP curve.

the payoff function (15). When such a function is explicitly given, the market clearing problems (14) can be omitted from problems (19)-(21).

\section{(2) Piecewise constant representation of the LMP curve}

Another difficulty that prevents problem (19)-21) from being efficiently solved is the product terms in the payoff function [15], which consist of the multiplication of LMP variables and energy quantity variables, because they are bilinear thus nonconvex. Our strategy is to approximate the LMP curve through a piecewise constant function which is expressed via binary variables, and then linearize each product term using integer programming techniques.

To this end, the feasible interval of demand is partitioned into $S-1$ segments (associated with binary variables $\beta_{s}, s=1, \cdots, S-1$ ) by $S$ breakpoints $p_{1}, \cdots, p_{S}$ (associated with continuous weight variables $\left.\alpha_{s}, s=1, \cdots, S\right)$. In the $s$-th interval between $p_{s}$ and $p_{s+1}$, the value of $\lambda$ is approximated by the arithmetic mean $\lambda_{s}=0.5\left[\lambda\left(p_{s}\right)+\lambda\left(p_{s+1}\right)\right]$, $s=1, \cdots, S-1$, as illustrated in Figure 6 Such a paradigm does not require a continuous assumption on the LMP curve, which is important because LMP could be discontinuous [61]. As a result, an arbitrary LMP-demand curve $\lambda(p)$ can be approximated by a piecewise constant function with an MILP-compatible form as follows,

$$
\begin{aligned}
& p=\sum_{s=1}^{S} \alpha_{s} p_{s}, \lambda=\sum_{s=1}^{S-1} \beta_{s} \lambda_{s} \\
& \alpha_{1} \leq \beta_{1}, \alpha_{S} \leq \beta_{S-1} \\
& \alpha_{s} \leq \beta_{s-1}+\beta_{s}, s=2, \cdots, S-1
\end{aligned}
$$




$$
\begin{aligned}
& \alpha_{s} \geq 0, s=1, \cdots, S, \sum_{s=1}^{S} \alpha_{s}=1 \\
& \beta_{s} \in\{0,1\}, s=1, \cdots, S-1, \sum_{s=1}^{S-1} \beta_{s}=1
\end{aligned}
$$

In 22, binary variable $\beta_{s}=1$ indicates interval $s$ is activated, and constraint 22e ensures that only one interval will be activated; Furthermore, constraints 22b - 22d enforce weight coefficients $\alpha_{s}, s=1, \cdots, S$ to be a special-ordered set of type-2, i.e., only adjacent two elements can be strictly positive, and their summation is 1 , whereas the rest are equal to 0 ; Finally, constraint 22a expresses $p$ and $\lambda$ via the linear combination of sampled values.

\section{(3) Linearizing the objective function}

The advantage of piecewise constant formulation (22) lies in the binary expression of $\lambda$, such that the product $\lambda \delta$ involving another continuous variable $\delta$ can be easily linearized via the integer programming technique. To see this, expand $\lambda$ using the formula in 22a, $\lambda \delta=\sum_{s=1}^{S-1} \beta_{s} \lambda_{s} \delta$, where $\beta_{s}$ is binary, $\lambda_{s}$ is constant, and $\delta$ is nonnegative and continuous. Let $\gamma_{s}=\beta_{s} \delta$, the product $\lambda \delta$ has a linear form $\sum_{s=1}^{S-1} \lambda_{s} \gamma_{s}$, and the relation among $\gamma_{s}, \beta_{s}$, and $\delta$ is captured by,

$$
0 \leq \delta-\gamma_{s} \leq M\left(1-\beta_{s}\right), 0 \leq \gamma_{s} \leq M \beta_{s}
$$

where $M$ is a constant which is greater than the value of $\delta$ at optimum. From 23], we can observe: whenever $\beta_{s}=0, \gamma_{s}=0$ is imposed by the second inequality, and the first constraint is redundant; otherwise, if $\beta_{s}=1, \gamma_{s}=\delta$ is enforced by the first inequality, and the second one is redundant. In either case, $\gamma_{s}=\beta_{s} \delta$ holds true.

Apply the above procedure to terms $\lambda_{t}^{e r} \delta_{i t}^{e-}, \lambda_{t}^{h r} \delta_{i t}^{h-}$ in energy hub payoff function (15), problems (19)- 21) can boil down to MILPs. An appealing feature is that the sizes of these MILPs are independent to the scale of the distribution system, since the exact AC power flow model has already been taken into account during the construction of the LMP curve. If the energy is connected to different buses, the situation will be much more complicated because we need an LMP surface with multiple inputs. Nevertheless, in the sharing market or the aggregation mode, energy hub should be geographically close to each other for the ease of energy delivery, so the proposed approach is appropriate in this special application. 
Remark 2:There are some optimization algorithms and software tools for nonlinear mixed integer programming, which might be used to solve this problem. In the literature, there have been some researches on the comparison of genetic algorithm (GA) based methods and MILP based methods. An improved real-coded genetic algorithm and an enhanced MILP based method were used to solve the unit commitment and economic dispatch of microgrids and compared in terms of computational time and accuracy in [62]. The results show that the MILP method needs less time to converge, while the GA method can achieve higher accuracy but is much more time costly. Admittedly, an improved GA method may solve our problem; however, as this paper mainly focus on economic efficiency difference among three organization schemes, so we just use the MILP approximation to solve it efficiently.

\section{Case Studies}

\subsection{System Configuration}

Numerical experiments were conducted on a modified IEEE 33-bus distribution system to validate the proposed model and theory. The topology of the test system is shown in Figure 7, the sites of 5 generators are marked in the same figure. The group of 30 energy hubs is connected to bus 3 . We consider a dispatch period of 4 hours. The day-ahead gas price $\lambda_{t}^{g c}$, day-ahead electricity price $\lambda_{t}^{e c}$, and real-time heat price $\lambda_{t}^{h r}$ are shown in Table 1. Other data of each energy hub are listed in Table 2 The load uncertainty is assumed to have a normal distribution. The predicted electric load in each period is [3.74, 3.75, 4.12, 3.73] MWh, and the predicted heat load in each period is $[2.18,2.25,3.45,2.89] \mathrm{MWh}$. The standard deviation is set as 0.2 . A fast-forward scenario reduction method based on the Kontorwish distance [63] is used for scenario selection. 5 typical scenarios are selected in the benchmark case. All the data of the test system can be found in [64]. MILP models were solved by CPLEX12.6 on a laptop with Intel(R) Core(TM) i7 CPU with $2.00 \mathrm{GHz}$ and $8 \mathrm{~GB}$ of RAM.

\subsection{Benchmark Case}

Numerical results for the market models under three organization schemes are listed in Table 3 . The computational time is acceptable in all the tests. The payoffs satisfy 


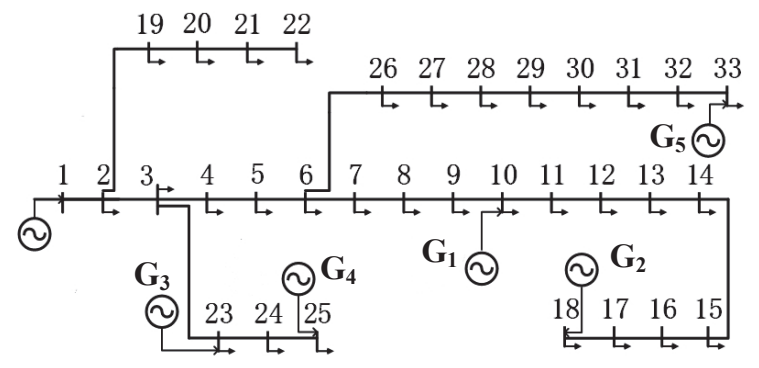

Figure 7: Topology of 33-node power system

Table 1: Energy prices (\$/MWh)

\begin{tabular}{ccccc}
\hline Period & 1 & 2 & 3 & 4 \\
\hline$\lambda_{t}^{g c}$ & 145.6 & 151.9 & 157.4 & 149.2 \\
$\lambda_{t}^{e c}$ & 264.9 & 270.6 & 272.2 & 262.4 \\
$\lambda_{t}^{h r}$ & 328.0 & 337.8 & 345.2 & 332.1 \\
\hline
\end{tabular}

Table 2: Energy hub data

\begin{tabular}{cccccc}
\hline Para. & MW & Para. & MW & Para. & no unit \\
\hline$p^{c h}$ & {$[0,2.0]$} & $E$ & {$[0,10]$} & $\eta_{e s}$ & 0.98 \\
$p^{\text {dis }}$ & {$[0,2.0]$} & $H$ & {$[0,10]$} & $\eta_{h s}$ & 0.98 \\
$h^{c h}$ & {$[0,2.0]$} & $\eta_{e h}$ & 0.98 & $\bar{I}$ & 5000 \\
$h^{\text {dis }}$ & {$[0,2.0]$} & $\eta_{g h}$ & 0.65 & $\eta_{g e}$ & 0.35 \\
\hline
\end{tabular}

$E C_{I D L}(=\$ 69194.11)>E C_{S M K}(=\$ 65528.38)>E C_{A G G}(=\$ 65267.25)$, which is consistent with Proposition 2 The total electric power demand and natural gas demand in the day-ahead market vary with time periods and depend on various factors, e.g., energy price, energy conversion efficiencies and storage capacities. For example, in period 3, although the day-ahead gas price is cheaper than the electricity price, energy hubs barely buy gas because of the low gas-to-power conversion efficiency; although the day-ahead electricity price in period 1 is cheaper than those in periods $2-3$, the contracted power in period 1 is zero. That is because the gas price in period 1 is low and the energy hubs can buy gas in period 1 , turning it into electricity and shifting it to other periods using energy storage units.

In addition, the MILP approximation accuracy can be adjusted by changing the 
Table 3: Results in the benchmark case

\begin{tabular}{cccc}
\hline & IDL & SMK & AGG \\
\hline Time (s) & 8.78 & 38.71 & 10.37 \\
expected cost (\$) & 69194.11 & 65528.38 & 65267.25 \\
$\sum_{i} p_{i 1}^{e 0}(\mathrm{MWh})$ & 0.00 & 0.00 & 0.00 \\
$\sum_{i} p_{i 2}^{e 0}(\mathrm{MWh})$ & 1.30 & 9.81 & 2.71 \\
$\sum_{i} p_{i 3}^{e 0}(\mathrm{MWh})$ & 72.45 & 65.98 & 69.65 \\
$\sum_{i} p_{i 4}^{e 0}(\mathrm{MWh})$ & 30.94 & 27.73 & 30.65 \\
$\sum_{i} p_{i 1}^{g 0} \mathrm{Sm}^{3} \mathrm{~h}$ & 466.07 & 466.32 & 466.32 \\
$\sum_{i} p_{i 2}^{g 0} \mathrm{Sm}^{3} \mathrm{~h}$ & 298.09 & 277.86 & 301.81 \\
$\sum_{i} p_{i 3}^{g 0} \mathrm{Sm}^{3} \mathrm{~h}$ & 0.00 & 14.23 & 0.00 \\
$\sum_{i} p_{i 4}^{g 0} \mathrm{Sm}^{3} \mathrm{~h}$ & 57.93 & 65.30 & 56.94 \\
\hline
\end{tabular}

Table 4: Optimal Values and computational time under different number of segments

\begin{tabular}{ccccccc}
\hline \multicolumn{2}{c}{ Segments } & 3 & 4 & 5 & 6 & 7 \\
\hline \multirow{2}{*}{ IDL } & Value(\$) & 69244.30 & 69194.11 & 69180.48 & 69186.68 & 69194.41 \\
& Time(s) & 11.72 & 8.78 & 213.46 & 525.35 & 879.59 \\
\hline \multirow{2}{*}{ SMK } & Value(\$) & 65562.82 & 65528.38 & 65530.41 & 65596.30 & 65546.54 \\
& Time(s) & 30.78 & 38.71 & 275.52 & 629.88 & 1933.72 \\
\hline \multirow{2}{*}{ AGG } & Value(\$) & 65319.86 & 65267.25 & 65254.62 & 65263.41 & 65271.85 \\
& Time(s) & 15.03 & 10.37 & 249.03 & 603.22 & 676.85 \\
\hline
\end{tabular}

number of segments in linearization. Table 4 shows that, the linearized model with 4 segments maintains a satisfactory balance between computational time and accuracy.

\subsection{Impact of Sampling and scenarios}

To examine the impact of random sampling and the fast-forward scenario reduction technique, we repeat the benchmark case 5 times. The average costs and relative standard deviation (RSD) are recorded in Table 5 Proposition 2 holds true in this set of tests. The RSD is always less than $2 \%$, indicating that the selected 5 scenarios can mimic the uncertainty with satisfactory accuracy. The average computation time is listed in Table 5 , confirming the efficiency of the proposed MILP solution approach.

Then, we test the impact of the number of scenarios on the market equilibrium. More scenarios are incorporated in our stochastic models [19]-21]. The costs and 
Table 5: Costs (\$) and computational time (s) under different samples

\begin{tabular}{ccccccc}
\hline Type & \multicolumn{2}{c}{ IDL } & \multicolumn{2}{c}{ SMK } & \multicolumn{2}{c}{ AGG } \\
\hline & Cost & Time & Cost & Time & Cost & Time \\
\hline 1 & 68659.9 & 7.73 & 65119.8 & 47.12 & 64853.6 & 12.26 \\
2 & 70440.9 & 10.59 & 66626.8 & 21.35 & 66363.4 & 11.78 \\
3 & 70227.9 & 11.52 & 67055.9 & 46.61 & 66842.3 & 16.52 \\
4 & 71661.3 & 9.72 & 68004.9 & 48.21 & 67740.1 & 14.89 \\
5 & 69194.1 & 8.78 & 65528.4 & 38.71 & 65267.3 & 10.37 \\
Avg. & 70036.8 & & 66467.1 & & 66213.3 & \\
RSD & $1.67 \%$ & & $1.75 \%$ & & $1.77 \%$ & \\
\hline
\end{tabular}

Table 6: Costs (\$) and computational time (s) under numbers of scenarios

\begin{tabular}{ccccccc}
\hline Type & \multicolumn{2}{c}{ IDL } & \multicolumn{2}{c}{ SMK } & \multicolumn{2}{c}{ AGG } \\
\hline & Cost & Time & Cost & Time & Cost & Time \\
\hline 5 & 69011.1 & 11.14 & 65936.6 & 43.44 & 65690.2 & 17.75 \\
6 & 68951.1 & 30.68 & 65769.8 & 57.06 & 65477.4 & 30.94 \\
7 & 69589.5 & 36.98 & 65858.6 & 67.27 & 65591.5 & 56.23 \\
8 & 68694.8 & 53.80 & 64996.9 & 113.24 & 64786.0 & 84.02 \\
9 & 69432.5 & 55.97 & 65944.3 & 208.87 & 65678.2 & 127.65 \\
\hline
\end{tabular}

computational time are listed in TABLE 6 The expected costs change little when more scenarios are included, showing that five scenarios are enough in this case. It takes longer time to solve the problem with the increasing number of scenarios, but the overall efficiency is satisfactory, validating the scalability of the proposed method.

\subsection{Impact of the Number of Energy Hubs}

The number of energy hubs is an pivotal factor that influences the performance of different organization schemes. The extreme case is that there is only one energy hub, in which the organization schemes are actually the same. We change the number of hubs from 10 to 60 , meanwhile, we maintain the total capacity of energy hubs unchanged to eliminate their influence on the real-time LMP and distribution system operation security. The expected costs in the three organization schemes are shown in Figure 8 , The differences between any two schemes are presented in Figure 9 


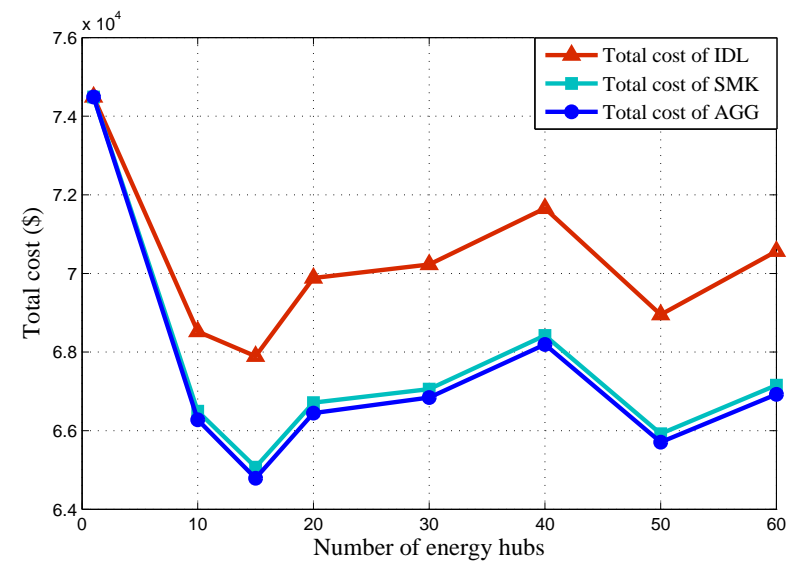

Figure 8: Costs under different numbers of energy hub.

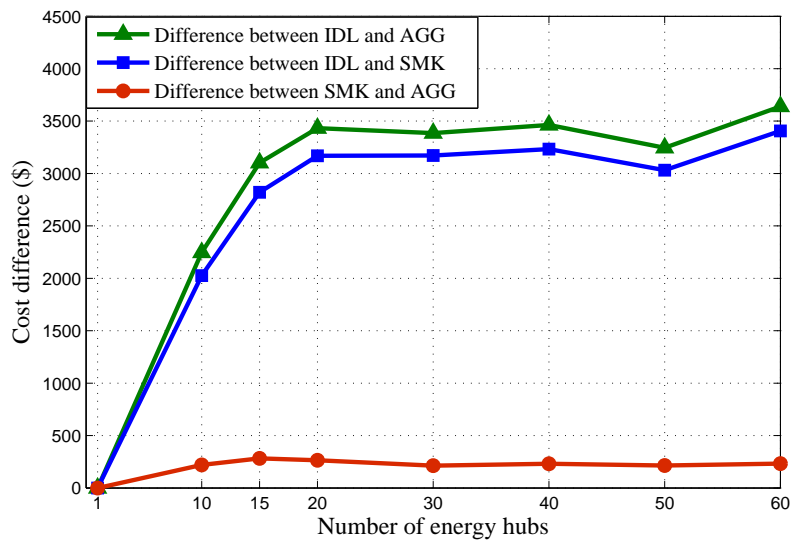

Figure 9: Cost Difference under different numbers of energy hub.

Several interesting phenomena are revealed. First, Proposition 2 always holds regardless of the number $N$ of energy hubs. Moreover, with the growth of $N$, the cost differences between any two organization schemes first increase and then tend to be constant as in Figure 9. This is because although the demand of a single energy hub is random, the total demand of the group of energy hubs may exhibit weaker volatility. For example, suppose when $N=1$, the expectation is $3 \mathrm{MW}$, and the variance is $0.04 \mathrm{MW}^{2}$, then if $N=50$, the expectation (variance) of each hubs is $0.06 \mathrm{MW}\left(1.6 \times 10^{-5} \mathrm{MW}^{2}\right)$; 
according to the Central Limit Theorem (or Large Number Law), the expected gross demand is still $3 \mathrm{MW}$, but the variance of gross demand is only $0.0008 \mathrm{MW}^{2}$. In reality, this means the more energy hubs involved, and smaller uncertainty the system needs to cope with. As a result, the cost differences change little when $N \geq 20$. The co-operation among larger number of market participants can bring more cost reduction.

\subsection{Impact of Uncertainty}

The degree of load uncertainty is another factor that greatly affects the costs of energy hubs. In this test, load uncertainty still follows the Normal distribution with expected values given in Subsection 5.1, and other system data are the same with the benchmark case. We change the standard deviation of the Normal distribution from 0 to $0.4 \mathrm{MW}$, the expected costs in the three organization schemes are shown in Figure 10.

When the standard deviation is equal to 0 , which means load is deterministic, all the energy hubs can sign exactly contracts in the day-ahead market and there is no need for real-time energy exchange as the day-ahead prices are always lower than the real-time energy prices. In such a circumstance, all three organization schemes share the same cost. With an increasing level of uncertainty, the expected costs grow accordingly. Nonetheless, when the uncertainty is small $(\sigma<0.2 \mathrm{MW})$, the expected costs in SMK and AGG scheme are scarcely influenced thanks to the energy exchange in the real-time stage. Even in the presence of large uncertainty, the cost growth rates in the SMK and AGG modes are smaller than that in the IDL mode due to the redistribution of energy in real time. This means that with a higher level of uncertainty, the cost differences between IDL and SMK, IDL and AGG rise, and thus the potential of cost reduction increases.

We can also find that, in the aforementioned cases, the cost differences between SMK and AGG are tiny, showing that the sharing market mode can acheive relatively high efficiency. Meanwhile, compared with the AGG mode, sharing market does not require a central coordinator, so that it is easier for application. Managing the energy hubs via the sharing market is promising. 


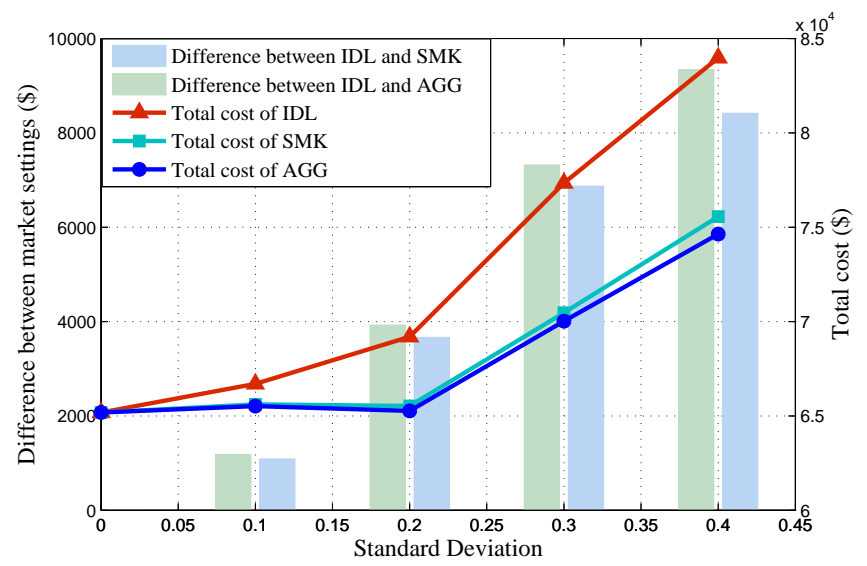

Figure 10: Costs under different standard deviation.

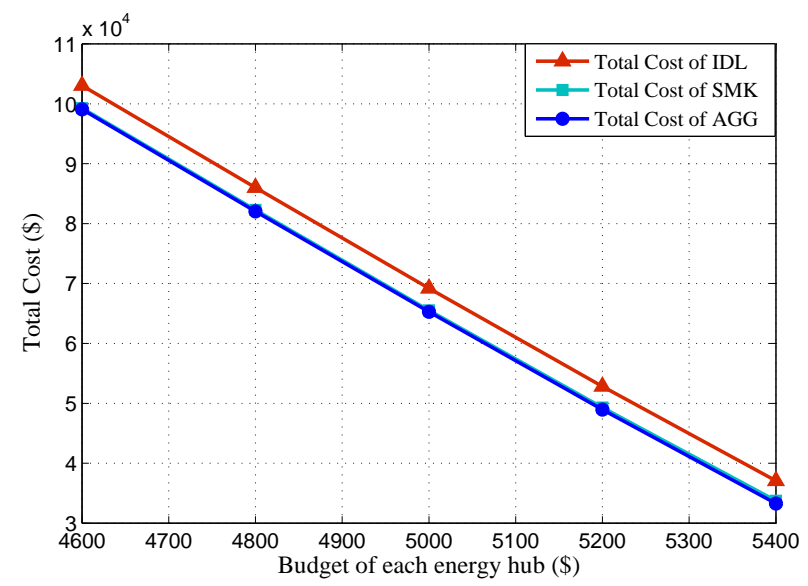

Figure 11: Costs under different budgets.

\subsection{Impact of Budget}

We change the budget of each energy hub from $\$ 4600$ to $\$ 5400$, and the expected costs in the three organization schemes are shown in Figure 11 , The relative difference between any two schemes are presented in Figure 12

When the budget $\bar{I}$ increases, the total cost of IDL scheme is larger than that of SMK scheme while the cost of AGG is always the least, which is in compliance with Proposition 2. From Figure 11, we can find that the costs under three schemes all de- 


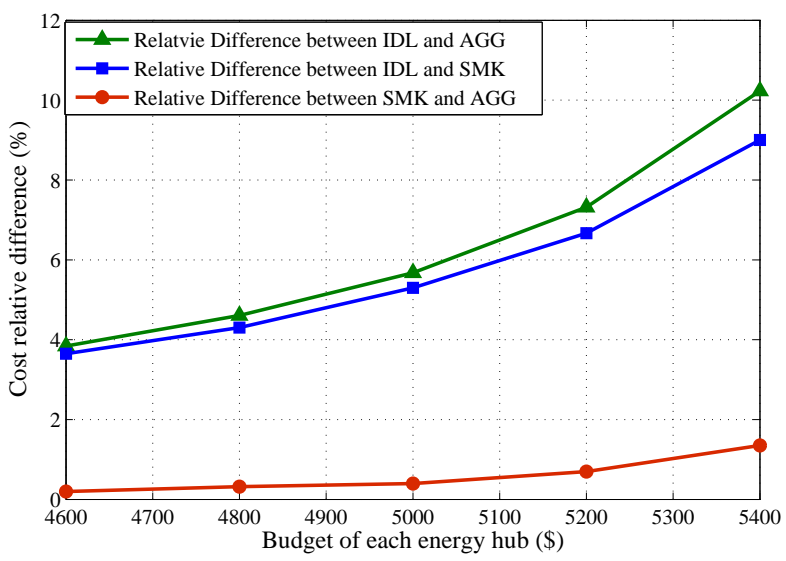

Figure 12: Cost relative differences under different budgets.

cline with the growth of $\bar{I}$. This is because when $\bar{I}$ rises, the energy hubs have larger feasible space in the day-ahead market and their flexibility in real time is also enhanced. In Figure 12, the relative differences between IDL and AGG, IDL and SMK increase rapidly while the relative difference between SMK and AGG grows moderately. When more budgets is available in the day-ahead market, more energies will be bought, enriching the deployable resource in the real-time stage. In this regard, SMK and AGG schemes can cut down more costs. The relative difference between SMK and AGG is tiny, showing that SMK scheme can achieve a high efficiency similar to AGG.

\subsection{Impact of Penalty Factor}

we multiply the penalty factor with a ratio varying from 0.5 to 1.5 and other parameters remain the same as the benchmark case. The expected costs in the three organization schemes are shown in Figure 13, The relative differences between any two schemes are presented in Figure 14

From Figure 13, we can find that Proposition 2 is always satisfied under different penalty factors. With the growth of penalty factor, the total costs in three market schemes all increase. The reason behind is straightforward: when the penalty factor is increasing, the extra costs for unfulfilled demand rise and so do the total costs. When the penalty cost accounts for a larger proportion of the total cost, the advantage of 


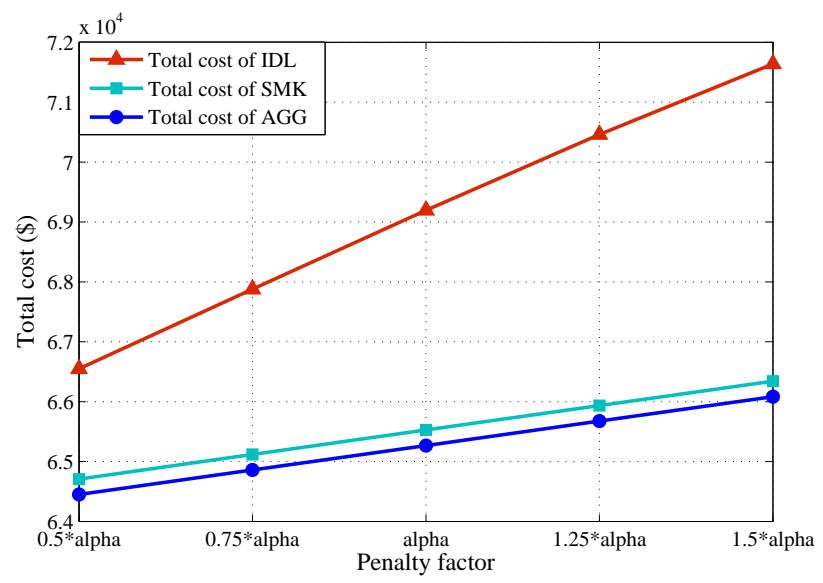

Figure 13: Costs under different penalty factors.

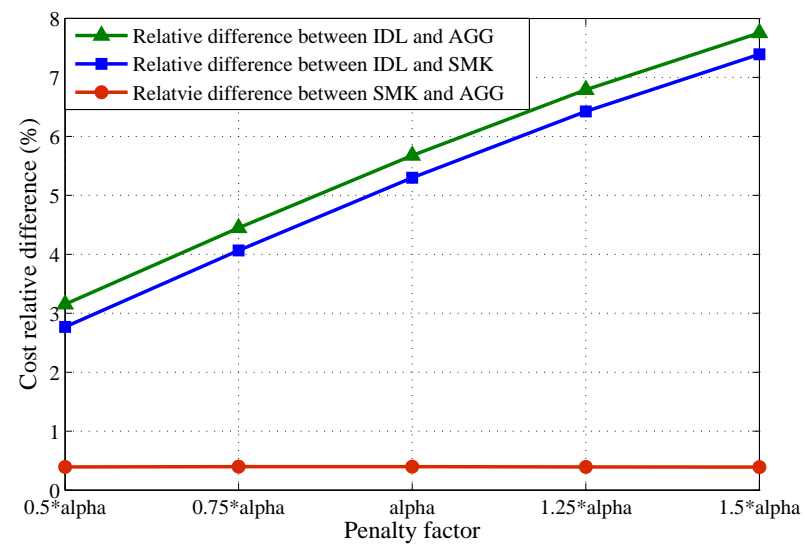

Figure 14: Cost relative differences under different penalty factors.

SMK and AGG schemes in cost reduction is more evident. We can see from Figure 14 that the relative differences between IDL and AGG, IDL and SMK increase with the penalty factor. Meanwhile, the relative difference between SMK and AGG is nearly constant, implying that under such penalty factors, the flexibility of SMK and AGG is fully exercised, and further increase in punishment cannot make a great difference.

Remark 3: The pool-market in the distribution system is organized by distribution system operator (DSO). The sharing market could be organized by a non-profit inter- 
mediary entity. We have concluded that the sharing market scheme can achieve nearly optimal efficiency without a central coordinator. Both the energy hubs and distribution system can benefit from our findings. If energy hubs implement the proposed method in a sharing market, they would have more flexibility in real-time and can reduce operation costs; for the distribution system, a certain fraction of demand uncertainty is mitigated in sharing market at the demand side, so the uncertainty faced by DSO is lessened.

\section{Conclusions}

Technologies of poly-generation are becoming increasingly mature nowadays. To promote the use of energy hubs, an effective business pattern is an important prerequisite. This paper presents a theoretical study on the efficiencies of individual, sharing, and aggregation schemes for managing a cluster of energy hubs with multiple energy demands. Under the individual scheme, each energy hub decides its own energy purchase plan without energy exchange with others. Under the sharing scheme, there is no central aggregator, each energy hub can decide its own energy purchase plan from the pool-market and also exchange energy with other hubs. Under the aggregation scheme, an aggregator collects the demand information from all energy hubs, signs day-ahead contracts on behalf of them and allocates energies to each hub in real-time. Utility curves of respective schemes are revealed, based on which the efficiencies are compared. The optimal energy exchange rate in the sharing scheme, which is called the price in the sharing market, is shown to be the day-ahead gas price to electricity price ratio. Concrete energy hub decision-marking models under each scheme are reformulated as MILP problems based upon duality theory, linearization techniques and theories of potential game and cooperative game. The optimal solution interprets the equilibrium in the market. Case studies convey the following information:

1) The aggregation scheme is the most efficient; the sharing market scheme has near optimal performance compared to the aggregation in various aspects, and requires no central coordinator, thus could be a promising direction in the future.

2) The performance of the sharing scheme depends on the number of energy hubs 
that take part in the market. The more, the better.

3) A proper designed penalty factor can fully exercise the flexibility of sharing without adding too much burden on each energy hubs.

To deploy sharing market scheme, an important task is to set up a platform (maybe electronic) which allows the clients to announce their needs and willingness to share. Sophisticated smart meters are also indispensable devices in the transition to the sharing economy. The analysis in this paper provides useful insights for designing policies for residential energy hubs and energy markets in the future. And the mathematical model in this paper could serve as the fundamental tool for studying a wide spectrum of market problems raised in multi-resource energy markets. Future research directions include the market power analysis as well as the market impact of sharing economy.

\section{Acknowledgement}

This work was supported in part by the National Natural Science Foundation of China (No. U1766203, No. 51677100, No. 51621065). The authors would like to thank Professor Antonio J. Conejo at Ohio State University for his helpful suggestions and insightful comments on this work.

\section{References}

[1] Wu, J., Yan, J., Desideri, U., Deconinck, G., Madsen, H., Huitema, G., et al. Synergies between energy supply networks. Appl Energy 2017;192:263-267.

[2] Muditha, A.. Combined analysis of coupled energy networks. Phd thesis, Cardiff University 2016;

[3] Li, H., Sun, Q., Zhang, Q., Wallin, F.. A review of the pricing mechanisms for district heating systems. Renew \& Sustain Energy Rev 2015;42:56-65.

[4] Experts discuss the central role cogeneration has to play in shaping EU energy policy, http://www.cogeneurope.eu/medialibrary/ $2011 / 04 / 21 / 2$ bc24419/24031120COGEN20Europe20press응 20 release.pdf 2011;COGEN, Europe. Available online. 
[5] Cogeneration in the European Union's energy supply security, http: //www. cogeneurope.eu/Downloadables/Publications / Cogeneration_Europe_Draft_paper_on_Security_of_ Supply_in_EU_energy_policy.pdf 2011;COGEN, Europe. Available online.

[6] Martinaitis, G.S.V., Andersen, A.N., Katz, J.. Feasibility of CHP-plants with thermal stores in the German spot market. Appl Energy 2009;86(11):2308-2316.

[7] IEA, . Energy policies of IEA countries: The United Kingdom 2002 review. International Energy Agency, Paris 2002;

[8] Combined heat and power-a clean energy solution, http://www . localpower.org/documents/chp_clean_energy_solution. pdf 2012;U.S. Department of Energy. Available online.

[9] Geidl, M., Andersson, G.. Optimal power flow of multiple energy carriers. IEEE Trans Power Syst 2007;22(1):145-155.

[10] Nuytten, T., Claessens, B., Paredis, K., Bael, J.V., Six, D.. Flexibility of a combined heat and power system with thermal energy storage for district heating. Appl Energy 2013;104(5):583-591.

[11] Wang, J.J., Jing, Y.Y., Zhang, C.F., Zhai, Z.. Performance comparison of combined cooling heating and power system in different operation modes. Appl Energy 2011;88(12):4621-4631.

[12] Noussan, M., Jarre, M., Roberto, R., Russolillo, D.. Combined vs separate heat and power production-primary energy comparison in high renewable share contexts. Appl Energy 2018;213:1-10.

[13] Amini, M.H., Boroojeni, K.G., Iyengar, S.S., Pardalos, P.M., Blaabjerg, F., Madni, A.M.. Sustainable interdependent networks: From theory to application. Springer 2018; 
[14] Caramanis, M., Bohn, R., Schweppe, F.. Optimal spot pricing: Practice and theory. IEEE Power Eng Rev 1982;PER-2(9):42-42.

[15] Li, F., Bo, R.. DCOPF-based LMP simulation: Algorithm, comparison with ACOPF, and sensitivity. IEEE Trans Power Syst 2007;22(4):1475-1485.

[16] Li, Y., Li, J., Wu, L.. Distribution system restructuring: Distribution LMP via unbalanced ACOPF. IEEE Trans Smart Grid in press 2017;.

[17] Overview of natural gas: Natural gas marketing, http://naturalgas. org/naturalgas/marketing/2013;NaturalGas.org. Avaiblable Online.

[18] Abada, I., Gabriel, S., Briat, V., Massol, O.. A generalized Nash-Cournot model for the Northwestern European natural gas markets with a fuel substitution demand function: The GaMMES model. Netw \& Spat Econ 2013;13(1):1-42.

[19] Gabriel, S.A., Conejo, A.J., Fuller, J.D., Hobbs, B.F., Ruiz, C.. Complementarity modeling in energy markets. Springer, New York 2013;.

[20] Chyong, K.C., Hobbs, B.F.. Strategic eurasian natural gas market model for energy security and policy analysis: Formulation and application to south stream. Energy Econ 2014;44:198-211.

[21] Setlhaolo, D., Sichilalu, S., Zhang, J.. Residential load management in an energy hub with heat pump water heater. Appl Energy 2017;208:551-560.

[22] Spiecker, S.. Modeling market power by natural gas producers and its impact on the power system. IEEE Trans Power Syst 2013;28(4):3737-3746.

[23] Chen, Y., Wei, W., Liu, F., Mei, S.. A multi-lateral trading model for coupled gas-heat-power energy networks. Appl Energy 2017;200:180-191.

[24] Wang, C., Wei, W., Wang, J., Liu, F., Mei, S.. Strategic offering and equilibrium in coupled gas and electricity markets. IEEE Trans Power Syst 2018;33(1):290-306. 
[25] Mather, J., Munsing, E.. Robust Cournot-Bertrand equilibria on power networks. American Control Conference 2017;:2747-2754.

[26] Bahrami, S., Amini, M.H.. A decentralized trading algorithm for an electricity market with generation uncertainty. Appl Energy 2018;218:520-532.

[27] Moiseeva, E., Hesamzadeh, M.R.. Bayesian and robust Nash equilibria in hydro-dominated systems under uncertainty. IEEE Trans Sustain Energy 2018;9(2):818-830.

[28] Carrion, M., Arroyo, J.M., Conejo, A.J.. A bilevel stochastic programming approach for retailer futures market trading. IEEE Trans Power Syst 2009;24(3):1446-1456.

[29] Hu, M.C., Lu, S.Y., Chen, Y.H.. Stochastic-multiobjective market equilibrium analysis of a demand response program in energy market under uncertainty. Appl Energy 2016;182:500-506.

[30] Kharrati, S., Kazemi, M., Ehsan, M.. Equilibria in the competitive retail electricity market considering uncertainty and risk management. Energy 2016;106:315328.

[31] Nojavan, S., Zare, K., Mohammadi-lvatloo, B.. Optimal stochastic energy management of retailer based on selling price determination under smart grid environment in the presence of demand response program. Appl Energy 2017;187:449464.

[32] Wei, W., Liu, F., Mei, S.. Energy pricing and dispatch for smart grid retailers under demand response and market price uncertainty. IEEE Trans Smart Grid 2017;6(3):1364-1374.

[33] Geidl, M., Keoppel, G., Favre-Perrod, P., Klockl, B.. Energy hubs for the future. Power \& Energy Magazine IEEE 2007;5(1):24-30.

[34] Geidl, M.. Integrated modeling and optimization of multi-carrier energy systems. PhD thesis, ETH Zurich 2007;. 
[35] Wasilewski, J.. Integrated modeling of microgrid for steady-state analysis using modified concept of multi-carrier energy hub. Int J of Electr Power \& Energy Syst 2015;73:891-898.

[36] Wang, Y., Cheng, J., Zhang, N., Kand, C.. Automatic and linearized modeling of energy hub and its flexibility analysis. Appl Energy 2018;211:705-714.

[37] Chicco, G., Mancarella, P.. A comprehensive approach to the characterization of trigeneration systems. In Proc of 6th World Energy System Conference, Turin, Italy 2006;.

[38] Hemmes, K., Zachariah-Wolf, J.L., Geidl, M., Andersson, G.. Towards multisource multi-product energy systems. International Journal of Hydrogen Energy 2007;32(10-11):1332-1338.

[39] Najafi, A., Falaghi, H., Contreras, J., Ramezani, M.. A stochastic bilevel model for the energy hub manager problem. IEEE Trans Smart Grid 2017;8(5):23942404.

[40] Yazdani-Damavandi, M., Neyestani, N., Shafie-Khah, M., Contreras, J., Catalao, J.P.S.. Strategic behavior of multi-energy players in electricity markets as aggregators of demand side resources using a bi-level approach. IEEE Trans Power Syst 2018;33(1):397-411.

[41] Chicco, G., Mancarella, P.. Matrix modelling of small-scale trigeneration systems and application to operational optimization. Energy 2009;34(3):261-273.

[42] Beccuti, G., Demiray, T., Batic, M., Tomasevic, N., Vranes, S.. Energy hub modelling and optimisation: an analytical case-study. PowerTech, 2015 IEEE Eindhoven IEEE 2015;:1-6.

[43] Yu, D., Lian, B., Dunn, R., Le, S.. Using control methods to model energy hub systems. Power Engineering Conference IEEE 2014;:1-4.

[44] Bahrami, S., Toulabi, M., Ranjbar, S., Moeini-Aghtaie, M., Ranjbar, A.M.. A decentralized energy management framework for energy hubs in dynamic pricing markets. IEEE Trans Smart Grid, early access 2017; 
[45] Samadi, P., Mohsenian-Rad, H., Schober, R., Wong, V.W.S.. Advanced demand side management for the future smart grid using mechanism design. IEEE Trans Smart Grid 2012;3:1170-1180.

[46] Deng, R., Yang, Z., Chow, M.Y., Chen, J.. A survey on demand response in smart grids: Mathematical models and approaches. IEEE Trans Ind Inf 2015;11(3):570-582.

[47] Park, S., Lee, J., Bae, S., Hwang, G., Choi, J.K.. Contribution-based energytrading mechanism in microgrids for future smart grid: A game theoretic approach. IEEE Trans Ind Electron 2016;63(7):4255-4265.

[48] Boyd, S., Vandenberghe, L.. Convex optimization. Cambridge University Press 2013;

[49] Najafi, A., Falaghi, H., Contreras, J., Ramezani, M.. Medium-term energy hub management subject to electricity price and wind uncertainty. Appl Energy $2016 ; 168: 418-433$.

[50] Najafi, A., Falaghi, H., Contreras, J., Ramezani, M.. A stochastic bilevel model for the energy hub manager problem. IEEE Trans Smart Grid 2017;8(5):23942404.

[51] Liu, N., Yu, X., Wang, C., Li, C., Ma, L., Lei, J.. Energy-sharing model with price-based demand response for microgrids of peer-to-peer prosumers. IEEE Trans Power Syst 2017;32(5):3569-3583.

[52] Sha, A., Aiello, M.. A novel strategy for optimising decentralised energy exchange for prosumers. Energies 2016;9(7):554.

[53] Damavandi, M.Y., Neyestani, N., Chicco, G., Shafie-khah, M., Catalao, J.P.S.. Aggregation of distributed energy resources under the concept of multi-energy players in local energy system. IEEE Trans Sustain Energy 2017;8(4):1679-1693.

[54] Farivar, M., Low, S.H.. Branch flow model: Relaxations and convexification. IEEE Trans Power Syst 2013;28(3):2554-2572. 
[55] Subhonmesh, B., Low, S.H., Chandy, K.M.. Equivalence of branch flow and bus injection models. Commun, Control, and Comput IEEE 2012;32(10):1893-1899.

[56] Zhao, Z., Wu, L., Song, G.. Convergence of volatile power markets with pricebased demand response. IEEE Trans Power Syst 2014;29(5):2107-2118.

[57] Monderer, D., Shapley, L.S.. Potential games. Games and Econ Behav 1996;14(1):124-143.

[58] Leyffer, S., Munson, T.. Solving multi-leader-follower games. Optim Methods $\&$ Softw 2010;25(4).

[59] Kamishiro, Y.. New approaches to cooperative game theory: Core and value. Brown University 2010;

[60] Gan, L., Li, N., Topcu, U., Low, S.H.. Exact convex relaxation of optimal power flow in distribution networks. Autom Control IEEE Trans 2015;60(1):7278.

[61] Li, F.. Continuous locational marginal pricing (CLMP). IEEE TransPower Syst 2007;22(4):1638-1646.

[62] Nemati, M., Braun, M., Tenbohlen, S.. Optimization of unit commitment and economic dispatch in microgrids based on genetic algorithm and mixed integer linear programming. Appl Energy 2018;210:944-963.

[63] Growe-Kuska, N., Heitsch, H., Romisch, W.. Scenario reduction and scenario tree construction for power management problems. Proc IEEE Bologna Power Tech Conf 2003;3:7.

[64] Data of test systems for multi-carrier energy systems with energy hubs, https://sites.google.com/site/yuechenthu/data-sheet 2018;Avaiblable Online. 